\title{
WestVirginiaUniversity
}

THE RESEARCH REPOSITORY @ WVU

Graduate Theses, Dissertations, and Problem Reports

2019

\section{Characterization of Major Flavonols in Ramps (Allium tricoccum)}

Wijdan M. Dabeek

West Virginia University, wmdabeek@mix.wvu.edu

Follow this and additional works at: https://researchrepository.wvu.edu/etd

Part of the Food Chemistry Commons, and the Other Nutrition Commons

\section{Recommended Citation}

Dabeek, Wijdan M., "Characterization of Major Flavonols in Ramps (Allium tricoccum)" (2019). Graduate Theses, Dissertations, and Problem Reports. 4020.

https://researchrepository.wvu.edu/etd/4020

This Thesis is protected by copyright and/or related rights. It has been brought to you by the The Research Repository @ WVU with permission from the rights-holder(s). You are free to use this Thesis in any way that is permitted by the copyright and related rights legislation that applies to your use. For other uses you must obtain permission from the rights-holder(s) directly, unless additional rights are indicated by a Creative Commons license in the record and/ or on the work itself. This Thesis has been accepted for inclusion in WVU Graduate Theses, Dissertations, and Problem Reports collection by an authorized administrator of The Research Repository @ WVU. For more information, please contact researchrepository@mail.wvu.edu. 


\title{
Characterization of Major Flavonols in Ramps (Allium tricoccum)
}

\author{
Wijdan M. Dabeek
}

Thesis submitted to the Davis College of Agriculture, Natural Resources, and Design at West Virginia University

in partial fulfillment of the requirements for the degree of

Master of Science

in Nutrition and Food Science
Melissa Ventura-Marra, PhD, RDN, Committee Chair
Callee Walsh, PhD
Nikola Kovinich, PhD

Department of Animal and Nutritional Sciences

Morgantown, West Virginia

2019

Key words: quercetin, kaempferol, ramps, glycosides, hydrolysis

Copyright 2019 Wijdan Dabeek 


\section{ABSTRACT \\ Characterization of Major Flavonols in Ramps (Allium tricoccum) \\ Wijdan M. Dabeek}

Ramps (Allium tricoccum) is a traditional plant in the eastern Appalachian Mountains. Ramps have been used in Appalachian folk medicine for its health promoting roles in lowering blood pressure and cholesterol. Information on the chemical composition of bioactive components responsible for the potential health benefits are scarce. Analysis of the chemical composition in similar Allium plants showed that flavonols, induced similar health benefits. Therefore, the aim of this work is to characterize major flavonol conjugates in ramps. The secondary aim is to develop a preliminary quantification protocol. Flavonol glycosides were extracted in 50\% methanol and 3\% acetic acid. Characterization of flavonol glycosides was conducted using UHPLC-PDA-MS². For acid hydrolysis protocol, flavonol glycosides extracts or plant tissues were hydrolyzed by different concentrations of HCl. UHPLC-PDA was used to detect hydrolyzed flavonols. Characterization showed that the major putative flavonol glycosides were kaempferol sophoroside glucuronide, quercetin sophoroside glucuronide, and quercetin dihexoside. The different parts of the putative structures were preliminarily confirmed using ${ }^{1} \mathrm{H}$ and $\mathrm{C}^{13}$ NMR analysis. The identified flavonol glycosides were concentrated in leaves. Preliminary quantification protocol showed that optimal hydrolysis conditions of flavonol conjugates was achieved by hydrolyzing the glycosides extract under 3M and 4M $\mathrm{HCl}$, respectively, at $90^{\circ} \mathrm{C}$ for two hours. To our knowledge, this work is the first to identify major flavonol glycosides content in ramps and develop a preliminary quantification protocol. This finding might explain the past usage of ramps as a medicinal plant. 


\section{ACKNOWLEDGMENTS}

This work wouldn’t be possible without the guidance and contribution of several people. First, I would like to express the deepest appreciation and gratitude to my committee chair, Dr. Melissa Ventura-Marra for her endless support during my educational journey. I am grateful for all the knowledge, experiences, passion, encouragement, and learning opportunities she provided. It was a great honor and pleasure to be a member of her research group. My words can’t thank her enough for gifting me an unforgettable and invaluable journey. Also, I would like to extend my gratitude to my committee members Dr. Nikola Kovinich and Dr. Callee Walsh for their help on each step of the project. I have gained an enormous amount of knowledge in a new advanced field of research from their experiences. I appreciate their time and efforts on this project.

My sincere thanks also go to Dr. Novruz Akhmedov for all the time, effort and expertise he provided on this work. His passion, assistance, and encouragement are invaluable. In a short period of time, he has provided a significant guidance and contribution on the project which are truly appreciated. I would also like to thank my research teammates for the help and all the insightful discussions. Also, I extend my appreciation to Denzil Blosser and Suzan Slider for all the help provided during ramps harvesting and processing.

Last but not least, I would like to extend my sincere and deepest gratitude and gratefulness to my supportive family. I would never have reached this stage or been the person I am today without their endless encouragement, attention, patience, and care. I greatly appreciate the time and advices they provided during all the ups and downs which have always inspired me to give my best. 


\section{TABLE OF CONTENT}

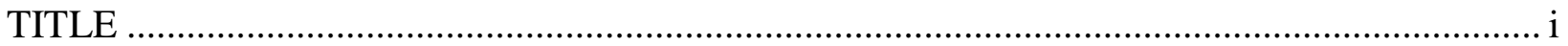

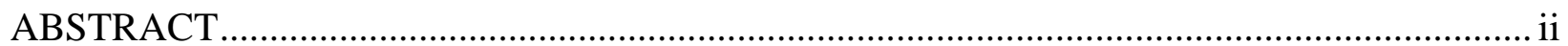

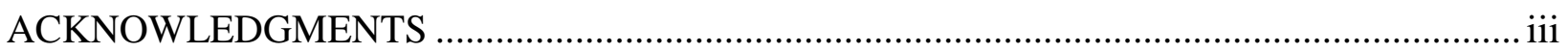

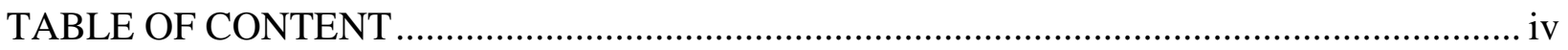

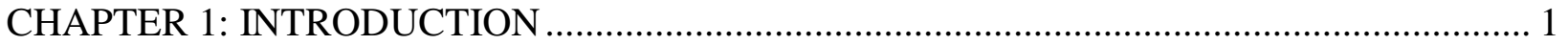

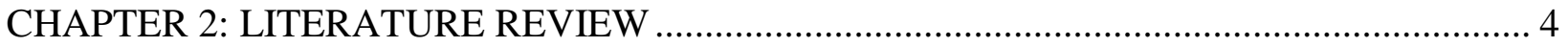

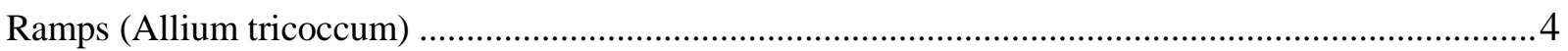

Chemical Composition of Allium Vegetables ......................................................................................

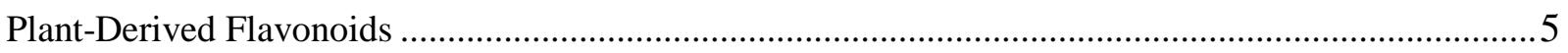

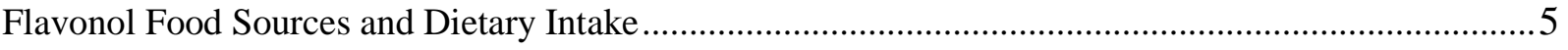

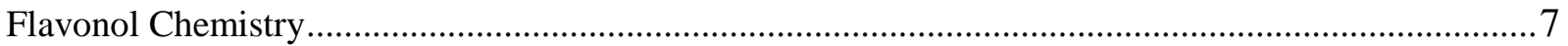

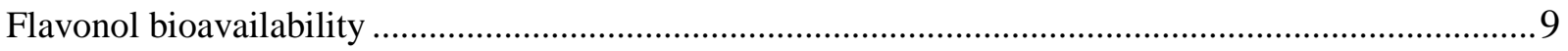

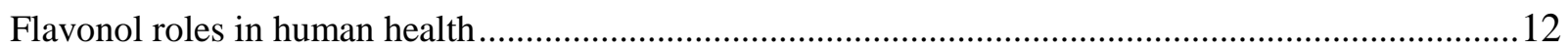

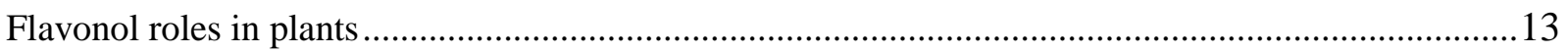

Extraction of plant flavonols.........................................................................................................14

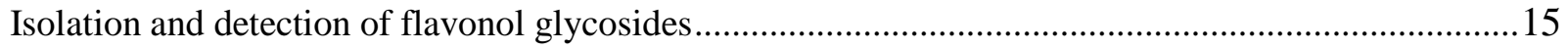

Structural characterization of flavonol glycosides............................................................................18

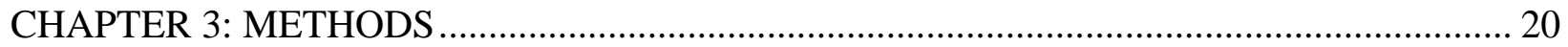

A.Separation and Characterization of Major Glycosides.................................................................22

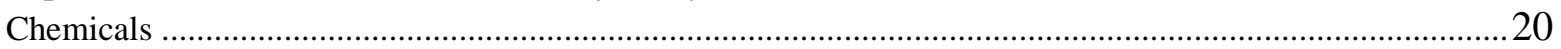

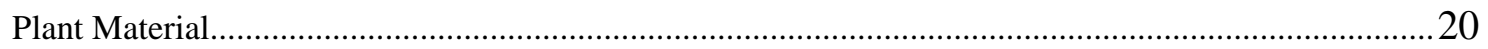

Extraction of Flavonols Glycosides ..................................................................................................2

Flavonol Glycoside Separation ......................................................................................................21

Characterization of Flavonol Glycosides by UHPLC-PDA-MS ${ }^{\mathrm{n}}$..........................................................22

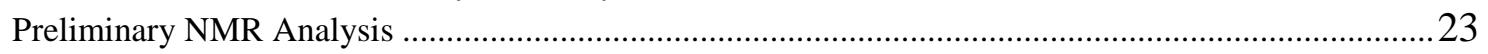

B.Preliminary Development of Acid Hydrolysis Protocol for Quantification of Flavonol Aglycones. .24

Preparation of Aglycone Standard Solutions ........................................................................................2

Development of Acid Hydrolysis Protocol .................................................................................................24

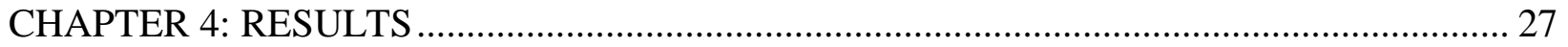

A.Separation and Characterization of Major Flavanol Glycosides....................................................2

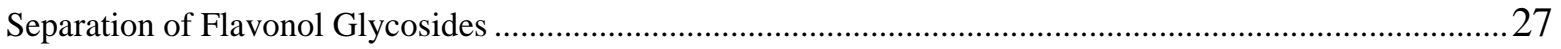

Characterization of Flavonol Glycosides ...............................................................................................2

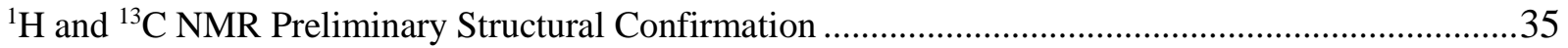


B.Preliminary Development of Acid Hydrolysis Protocol for Quantification of Flavonol Aglycones ..38

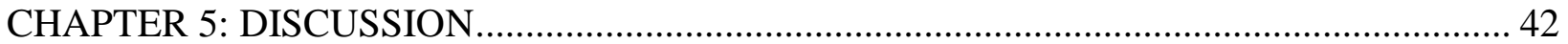

A.Separation and Characterization of Major Flavanol Glycosides................................................42

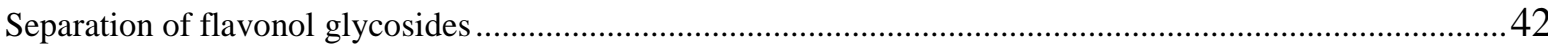

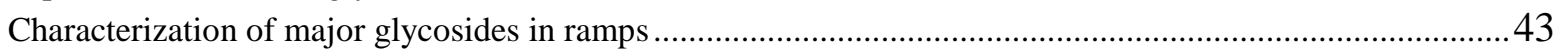

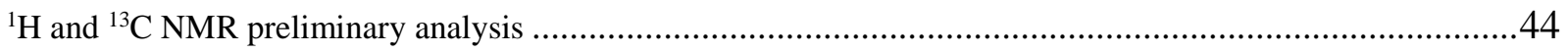

B. Preliminary Development of Acid Hydrolysis Protocol for Quantification of Flavonol Aglycones .45

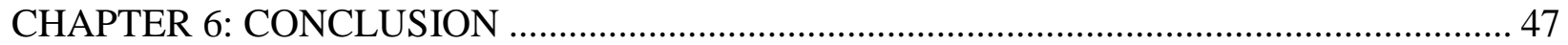

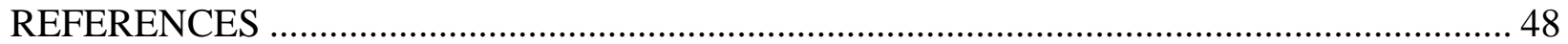




\section{CHAPTER 1: INTRODUCTION}

Allium tricoccum, known as ramps or wild leek, is a spring vegetable native to the eastern Appalachian mountains in the United States (U.S) [1]. Ramp is a member of the allium vegetables that include wild garlic, leek, onion, and garlic [2]. This bulb-forming plant has broad green leaves, purple stalk, and white bulb [3]. Ramp is the first green to grow as patches in rich, moist shady woods from north to Canada, west to Missouri and Minnesota, and south to North Carolina [4,5]. The Cherokee Indians in the U.S. consumed ramps as a medicinal plant to treat for colds and earache, and as a spring tonic [6]. In addition, folk medicine in Appalachian mountains recommends ramps for lowering blood pressure, lipids, and cholesterol [6,7].

Although the chemical composition and bioactive components of ramps are unknown, its garlic-like odor suggested that it contains sulfur compounds, the volatile components in allium vegetables [8]. One study identified sulfur compounds in ramps' bulb and reported that thiosulfinates and allicin were major sulfur components in ramps [8]. Another study reported that ramp's leaves contain high amount of vitamin C [9]. However, there are no studies on the characterization or quantification of other bioactive components that might induce the reported health benefits of ramps. Previous studies investigating the health benefits of closely related Allium vegetables, such as wild garlic and leeks, reported blood pressure and cholesterol-lowering effects when fed to rats [10,11]. Flavonoids, large group of plant polyphenols, was reported as one of the major bioactive components to provide the medicinal benefits of allium vegetables [12]. Flavonoids are plant pigments and secondary metabolites synthesized by the phenylpropanoid pathway [13]. The flavonoids in plants are found in complex conjugates, with different sugar moieties linked to the aglycone backbone known as glycosides [14]. Flavonoids are categorized 
under six classes including flavanones, flavanols, isoflavones, flavan-3-ols, flavonols, and anthocyanins [15].

Flavonols including kaempferol, quercetin, and myricetin, is the most ubiquitous class in fruits and vegetables, specifically, allium vegetables $[3,16,17]$. The United States Department of Agriculture (USDA) flavonoids database reported that flavonol was the only class detected in leeks and spring onions which are allium vegetables closely related to ramps [18]. The quantity of flavonols in plants varies by season, light and degree of ripeness [19-22]. Additionally, individual flavonols are unequally distributed in plant parts with different pigments [23]. Quercetin but not kaempferol was detected in the bulb of red spring onion [23]. However, a lower amount of quercetin, and kaempferol were detected in the leaf of red spring onion and high amounts of kaempferol were found in spinach [23]. Therefore, kaempferol is rich in the green leafy plant parts while quercetin is concentrated in parts close to roots and bulbs [24,25].

It has been suggested that the rates of glycoside digestion and absorption differ depending on the sugar moiety attached to flavonols in plants, [26]. Although quantifying flavonols in plants is important, higher flavonols content doesn’t reflect higher absorption and biological activity in humans [26,27]. Thus, to understand the absorption and bioactivity of flavonols in the human body, it is essential to characterize the glycoside conjugates. The major flavonol glycosides in red onion include quercetin-4'-glucoside and quercetin-3,4'-diglucoside [28]. In wild garlic kaempferol glycosides in the form of kaempferol 3-O-beta-glucopyranoside and kaempferol 3-Obeta-neohesperidoside have been detected [3]. However, the characterization and quantity of flavonol glycosides in ramp plants are unknown.

To better understand the potential mechanism health benefits of ramps, characterization of flavonol glycosides in whole ramps and individual parts is needed. Therefore, the primary 
objective of this study is to characterize flavonol glycosides in whole ramps and the individual parts (i.e. leaf, stem and bulb). The secondary objective is to a develop preliminary acid hydrolysis protocol that could be used to quantify total flavonol aglycones in ramps. 


\section{CHAPTER 2: LITERATURE REVIEW}

\section{Ramps (Allium tricoccum)}

Ramps (Allium tricoccum) is native to deciduous temperate forests in the eastern Appalachian Mountains in the U.S. [1]. After a long winter season, the snow melts and soil temperature increases providing optimal growing condition for ramps [5]. To fully mature, ramp seeds can take 5 to 7 years [5]. There are two varieties of ramps, variety tricoccum and burdickii [5]. The triccocum variety is dominant in the southern Appalachian part and has a larger leaf with purple stem. Burdickii variety, however, is more common in the north and has narrower leaves with no pigmented stem.

Ramps are known as traditional and local seasonal food in Appalachia [29]. American Indians consumed ramps as a tonic and to provide vitamins and minerals after winter season because it is the first green to grow [6]. Ramp leaves were considered a folk remedy to cleanse the blood by reducing blood cholesterol and lipids [7]. However, the reported health benefits have not been proven in clinical trials. Also, reports on the nutrient composition of ramps are limited. Studies on similar allium vegetables, i.e. leeks and wild garlic, report potential cardioprotective and cholesterol-lowering effects $[10,11]$. In order to analyze the chemical composition of bioactive compounds in ramps, it is important to understand the chemical composition of other allium vegetables.

\section{Chemical Composition of Allium Vegetables}

Calvey et al analyzed organosulfur compounds in ramps harvested from three geographic regions Quebec in Canada, Potomac River and West Virginia in the U.S [8]. Results suggested that thiosulfonates were the major sulfur compounds followed by allicin in ramps harvested from 
the Potomac River region and West Virginia (5-7 and $3 \mu \mathrm{mol} / \mathrm{g}$, respectively) [8]. In another study vitamin C (0.8 mg/g dry weight) was detected in ramps harvested from southern Ohio and northern Kentucky in the U.S [9]. Analysis of nutrients and bioactive compounds in ramps is still needed.

To determine potential nutrients and bioactive components in ramps, the chemical composition of allium plants closely related to ramps (i.e.leeks and wild garlic) [8], were reviewed. Analysis of the bioactive substances indicated high levels of flavonoids; 35.14 and $41.01 \mathrm{mg} / \mathrm{g}$ dry weight in leeks and wild garlic, respectively [30,31]. The United States Department of Agriculture (USDA) flavonoids database reports flavonols as the only flavonoid subclass in leeks and spring onions [18]. Therefore, it is important to understand the major characteristics and detection methods of flavonols.

\section{Plant-Derived Flavonoids}

Flavonoids are plant secondary metabolites. That is, they are chemicals not required for the growth and development of the plant but are synthesized to enhance plant survival in its environment [32]. Plant flavonoids also play an important role as active pharmaceuticals [32]. In plants, flavonoids are responsible for the different pigments and aroma [33]. Due to the complexity and variation in food sources and chemical structure, flavonoids are classified by subclasses, including flavonols, flavanones, isoflavones, flavones, flavan-3-ol, and anthocyanins [17]. Flavonols are the most ubiquitous flavonoid subclass in fruits and vegetables, specifically allium vegetables $[3,16,17]$.

\section{Flavonol Food Sources and Dietary Intake}

Flavonols are widely distributed in fruits and vegetables [33]. Table 1 shows the average amounts of quercetin and kaempferol in major food sources. Quercetin is high in a few plant 
foods including capers, onion, asparagus, and berries; but, found in small amounts in many fruits and vegetables. The richest plant sources of kaempferol are green leafy vegetables, including spinach, kale, and herbs like dill, chives, and tarragon.

The estimated amounts of flavonols intake within a population vary. This variation is related, in part, to databases completeness. Currently, there is no complete standard database on flavonoids content in foods. The three databases available are United States Department of Agriculture (USDA) database for flavonoids [18], eBasis (BioActive Substances in Food Information System) database [38], and Phenol-Explorer [35]. Additionally, the amount of flavonols in plants vary by season, light, and degree of ripeness [19-21]. Due to these limitations in estimating flavonols intakes in a population, dietary recommendations of flavonols intake for individuals has not been established [36,37]. Despite being widely available in foods, flavonols intake in the U.S. range between 9-36.2 $\mathrm{mg} /$ day [38] which is lower than flavonols intake in the United Kingdom and European countries (average 51 and $52 \mathrm{mg} /$ day, respectively) [39,40]. In the U.S., the highest sources of flavonols were tea, onion, apple, and red wine [41,42]. Average intakes of individual quercetin and kaempferol among U.S. adults is 13.5 and 5.4 mg/day, respectively [41]. 
Table 1. Selected Plant Sources of Quercetin and Kaempferol

\begin{tabular}{|c|c|c|}
\hline Plant & Quercetin & Kaempferol \\
\hline & \multicolumn{2}{|c|}{$\mathrm{mg} / 100 \mathrm{~g}$ fresh weight } \\
\hline \multicolumn{3}{|l|}{ Vegetables } \\
\hline Asparagus ${ }^{b}$ & 14.0 & 1.40 \\
\hline Broccoli $^{\text {a }}$ & 13.7 & 7.20 \\
\hline Capers (raw) ${ }^{\text {b }}$ & 234 & 259 \\
\hline Chili pepper ${ }^{\text {a }}$ & 32.6 & - \\
\hline Chinese cabbage $^{\mathrm{b}}$ & - & 22.5 \\
\hline Ginger ${ }^{b}$ & - & 33.6 \\
\hline Kale $^{\text {b }}$ & 22.6 & 47.0 \\
\hline Leeks [30] & 2.5 & 11.8 \\
\hline Lettuce $^{a}$ & 14.7 & 0.84 \\
\hline Onions ${ }^{a}$ & 45.0 & 4.50 \\
\hline Shallot [43] & 0.34 & 11.7 \\
\hline Spinach $^{\mathrm{a}}$ & 27.2 & 55.0 \\
\hline Tomatoes $^{\text {a }}$ & 7.40 & 0.84 \\
\hline \multicolumn{3}{|l|}{ Herbs/Spices } \\
\hline Chives $^{\mathrm{a}}$ & 10.4 & 12.5 \\
\hline Dill $^{a}$ & 79.0 & 40.0 \\
\hline Fennel leaves $^{a}$ & 46.8 & 6.50 \\
\hline Oregano $^{b}$ & 42.0 & - \\
\hline Terragon ${ }^{b}$ & 10.0 & 11.0 \\
\hline \multicolumn{3}{|l|}{ Fruits } \\
\hline Apples b & 4.01 & 0.14 \\
\hline Blueberry $^{a}$ & 14.6 & 3.17 \\
\hline Cherry $^{\mathrm{b}}$ & 17.4 & 5.14 \\
\hline Cranberry $^{a}$ & 25.0 & 0.21 \\
\hline & \multicolumn{2}{|c|}{$\mathrm{mg} / 100 \mathrm{ml}$} \\
\hline Beverages & & \\
\hline Black tea $^{a}$ & 2.50 & 1.70 \\
\hline Green tea $^{\text {a }}$ & 2.38 & 1.50 \\
\hline Red wine $^{\mathrm{a}}$ & 3.16 & 0.25 \\
\hline
\end{tabular}

${ }^{\text {a }}$ Phenol-Explorer: database for polyphenol content in foods.

${ }^{\mathrm{b}}$ USDA database for the flavonoid content of selected foods.

\section{Flavonol Chemistry}

Chemically, flavonoids consist of two phenyl rings (ring A and B) connected to a heterocyclic ring (ring C) [14]. The flavonol subclass share the same 3-hydroxy flavone backbone but differ in the presence and position of two hydroxyl groups at R1 and R2 [44]. Figure 2 shows the general chemical structure of flavonols. The number of hydroxyl groups on the structure determines chemical stability and reactivity [44]. It was suggested that the 
hydroxyl groups on B and C rings provide anti-oxidant and free-radical scavenging function [44]. Kaempferol is the most chemically stable flavonol as it has fewer hydroxyl groups.

Flavonols in their free form, aglycones, have lipophilic (fat-soluble) properties. However, most flavonols are synthesized in plants attached to a sugar moiety known as glycosides [45]. Some of the sugar moieties include the monosaccharides glucose, rhamnose, galactose, arabinose, and xylose [46]. Additionally, the sugar moiety can be a disaccharide such as a rutinoside, composed of glucose and rhamnose connected by $\beta$ glycosidic bond [45]. The hydroxyl functional groups on all three rings are potential sites for links to sugar moieties know as O-glycosides [14]. The glycoside attachment converts the lipophilic flavonol to a lipophobic (water-soluble) form. Consequently, flavonol glycosides have different polarity and rates of digestion and absorption in the human body compared to the aglycone form [47].<smiles>[R]c1cc(-c2oc3cc(O)cc(O)c3c(=O)c2O)cc([R2])c1O</smiles>

\begin{tabular}{ccc}
\hline Flavonol & $\mathbf{R}_{1}$ & $\mathbf{R}_{2}$ \\
\hline Kaempferol & $\mathrm{H}$ & $\mathrm{H}$ \\
\hline Quercetin & $\mathrm{H}$ & $\mathrm{OH}$ \\
\hline
\end{tabular}

Figure 1. General chemical structure of quercetin and kaempferol. Two phenyl rings (A and B) and heterocyclic ring (C). 


\section{Flavonol Bioavailability}

In order to exert a beneficial effect on health, ingested flavonols need to be bioavailable to body tissues. The definition of bioavailability varies depending on the form of the ingested compound. Bioavailability of pharmaceutical drugs refers to the extent and rate of digestion and absorption to which the active compound becomes available at the site of action [48]. The bioavailability from a nutritional perspective, however, is defined as the extent of digestion, absorption, metabolism, and excretion after the ingestion of foods [49]. Figure 2 illustrates the general overview of food-derived flavonol bioavailability, including digestion, transport across the intestinal barrier into the blood, distribution to body tissues, metabolism, and excretion. One of the important factors of bioavailability is the fat solubility of the ingested flavonols [49].

When flavonol-rich foods are consumed, the aglycone and glycoside forms undergo different routes of digestion and absorption [22]. Lipophilic aglycones passively diffuse unmetabolized from the intestinal lumen into the enterocytes where they are either directly absorbed into the hepatic portal vein or metabolized before absorption [22,50]. Metabolism of the aglycones in the enterocytes involves phase I (oxidation and O-demethylation) and phase II metabolism (sulfation, glucuronidation, and methylation) producing lipophobic metabolites which are absorbed via ATP-binding cassette (ABC) transporters into the hepatic portal vein [50,51]. Lipophobic glycosides, however, must be hydrolyzed to the aglycone form before absorption into the blood [52]. The hydrolysis of flavonol glycosides depends on the type of sugar attached to the aglycone backbone [53]. In general, flavonol glycosides are hydrolyzed either in the intestinal lumen or enterocyte. On the intestinal brush border, lactase-phlorizin hydrolase enzyme (LPH) hydrolyzes glycosides producing aglycones in the intestinal lumen [54]. Hydrolyzed aglycones are then passively absorbed into enterocytes. Alternatively, the glycosides can be transported by 
sodium-dependent glucose transporter (SGLT 1) into the enterocyte, where they are hydrolyzed to aglycones by cytosolic $\beta$-glucosidase [52,55]. Resulting aglycones in the enterocytes either passively diffuse into the hepatic portal vein or undergo phase I \& II metabolism producing metabolites which are absorbed via ABC transporters [50,51].

After intestinal digestion and absorption, the aglycones bind to serum albumin and the flavonol metabolites are transported via the hepatic portal vein to the liver [56]. In the liver, the remaining aglycones undergo phase I \& II metabolism resulting in methyl, sulfur, and glucuronide metabolites which are transported along with intestinal metabolites into the systemic circulation for distribution to body tissues [57,58]. Although flavonols' metabolism in body tissues is not well understood, an in vitro study reported that conjugated metabolites in body tissues could be converted back to aglycones by $\beta$-glucuronidase resulting in the accumulation of flavonol aglycone in body tissues [59]. Excretion of flavonol metabolites occurs by urinary and biliary elimination. Metabolites in bile are either eliminated in feces or recycled back to the small intestine [15]. Excreted metabolites may differ from those in the blood indicating potential metabolism in the kidneys and body tissues [60].

Some ingested glycosides bypass the small intestine and reach the colon where the colonic microbiome metabolism occur [58]. Colonic metabolism includes the hydrolysis of glycosides resulting in aglycones which undergo ring fission producing phenolic acid metabolites [61]. The major metabolites produced are 3,4-dihydroxyphenylacetic acid, 3,4-dihydroxybenzoic acid, and 3-hydroxyphenylacetic acid [62]. These metabolites are either excreted in feces or absorbed into the circulation [80]. The fate and bioactivity of phenolic acid metabolites are not well understood. Absorbed flavonols reach body tissues to exert benefits, mainly, cardioprotective, and antioxidant effects. 


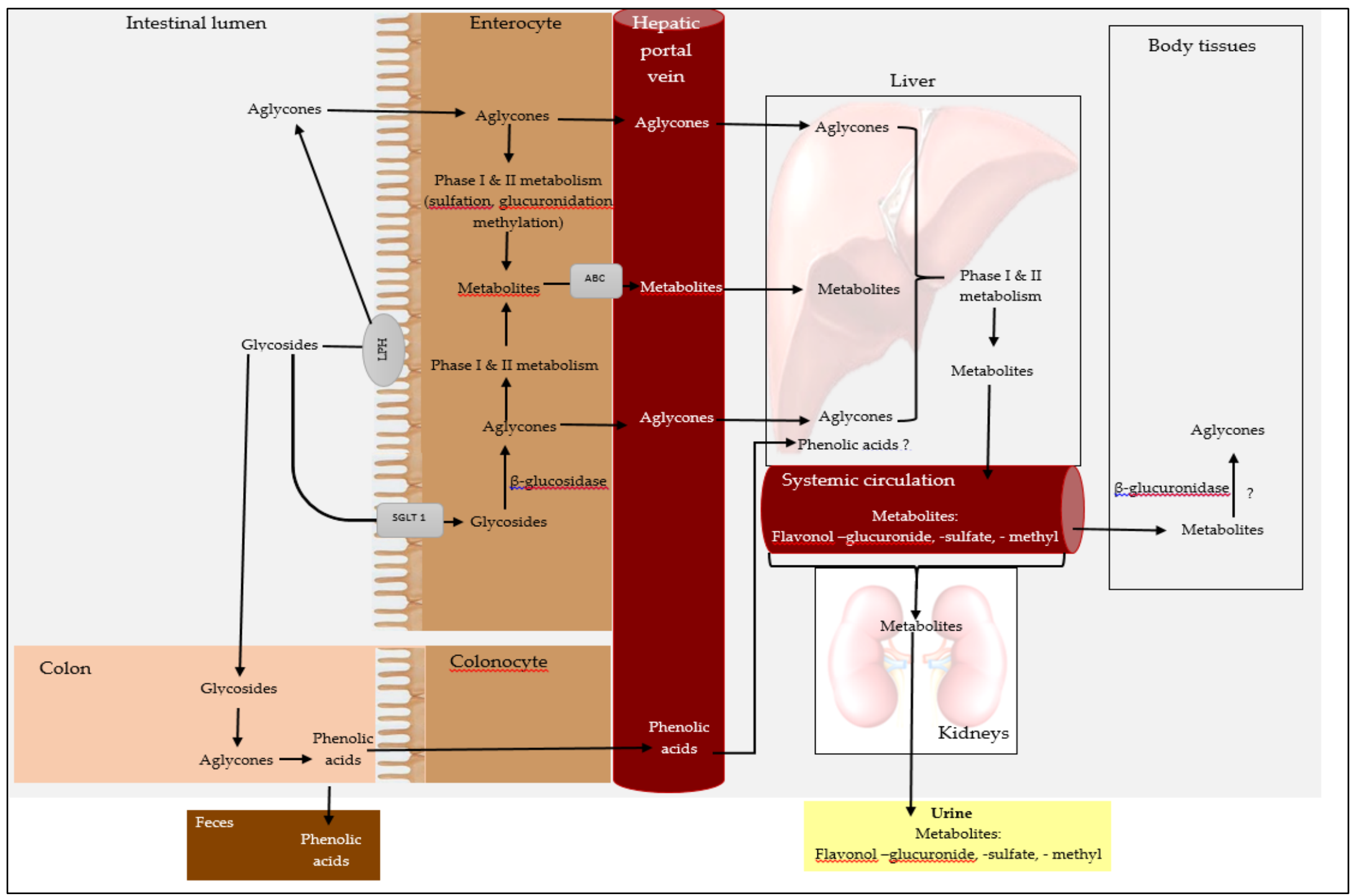

Figure 2. General overview of food-derived flavonol bioavailability.

Abbreviations: LPH, lactase-phlorizin hydrolase enzyme; SGLT 1, sodium-dependent glucose transporter; ?, mechanism is not well understood. 


\section{Flavonol Roles In Human Health}

The bioactivity of quercetin was confirmed in vitro studies, which suggested that quercetin has cardiovascular and antihypertensive benefits due to its antioxidant properties [64]. Furthermore, quercetin has been shown to act as an anti-inflammatory agent on cultured cells and ex vivo [65]. Also, clinical trials investigating the bioactivity of quercetin on blood pressure and cardiovascular risk reported potential cardioprotective effects.

Studies on hypertensive patients examined the effectiveness of quercetin in lowering blood pressure. A dose of $150 \mathrm{mg}$ quercetin supplement was effective in reducing blood pressure in hypertensive patients after 42 days of treatment [66]. Also, a dose of $730 \mathrm{mg}$ quercetin supplement was also effective in reducing blood pressure in patients diagnosed with stage 1 hypertension but not in pre-hypertensive individuals [67].

The potential cardioprotective effects of kaempferol are related to its anti-oxidant and anti-inflammatory activity reported in vitro and animal studies [68-70]. It has been suggested that kaempferol reduces reactive oxygen species (ROS) due to its free-radical scavenging role, which prevents tissue damage and inflammation [71,72]. Clinical studies on the cardioprotective benefits of kaempferol are limited. Epidemiological studies, however, investigated the association between high intake of dietary flavonoids, specifically kaempferol, and cardiovascular health. Assessment of the association between the intake of individual flavonols and myocardial infarction (MI) and fatal coronary heart disease (CHD) in Nurse' Health cohort (66,360 women), reported that kaempferol intake, mainly from broccoli and tea, was inversely associated with CHD with a relative risk of $0.66(95 \% \mathrm{CI}: 0.48-0.93, \mathrm{p}=0.04)$ but no significant association was observed for MI [73]. However, two epidemiological studies reported a 
significant negative association between acute and fatal MI with higher flavonol and kaempferol intake $[74,75]$.

\section{Flavonol Roles In Plants}

Flavonols are ubiquitously found in plants mainly as glycoside conjugates in the vacuoles of plant cells [76]. In addition to other major pigments, carotenoids, and chlorophyll, flavonols provide color pigments in fruits, vegetables, and flowers which attract pollinating insects [77]. Flavonoids, including flavonols, function as signaling molecules to protect plant tissues from ultraviolet (UV) radiation [78]. Due to their antioxidant capacity, flavonols function as detoxifying agents in plants [77]. Flavonols neutralize byproducts of free radicals and oxygen species produced as a result of photosynthesis and other biological reactions in plants [77].

Abiotic stress in plant refers to stress induced by non-living factors including temperature, water availability, heat, and composition of the soil. Although flavonols function under abiotic stress is still unclear, a study found that plants exposed to abiotic stress conditions that caused an increase in oxidative damage will have a higher synthesis rate of flavonols [79]. Also, under cold temperature, flavonols content specifically quercetin, increases to provide antioxidant protection of plant tissues [80].

To investigate the benefits of the particular flavonol-rich plant, it is essential to develop an analysis protocol for the detection of flavonols in plants. Analytical methods for the characterization of plant-derived flavonols varies depending on individual plant species as they synthesize different flavonol conjugates [13]. Thus, understanding the steps of flavonols detection from different plants are required for the analytical method development. 


\section{Extraction of Plant Flavonols}

The first step in the identification of a bioactive compound in plant is extraction. The process of extraction is performed by separating the desired compounds from plant tissues, which eliminate the interference of other plant components [81]. To extract bioactive compounds, plant material is mixed in a solution with specific polarity based on the chemical characteristics of the desired compounds [81]. Water soluble compounds, lipophobic, are extracted in a polar solution while fat soluble, lipophilic, compounds are extracted in a non-polar solution.

Polyphenols extraction involves the optimization of several factors, including the selection of extraction solution, and the particle size of the extracted plant material [82]. The solvents are selected based on the solubility of the desired bioactive components, which lead to the extraction of substances soluble in the selected solvent leaving the insoluble material. Although flavonol's chemical structure indicated lipophilic properties, in plants, the attachment of sugar moieties decreases lipophilicity (increases polarity) of flavonols, and thus, it impacts the solubility of plant-derived flavonols. Diem Do et al. [83] examined the effects of different extraction solvents on the maximum content of flavonoids extracted from the medicinal plant Defatted L. aromatica. Extraction solvents were 100\% water, methanol (100\%, 75\%, and 50\% in water), ethanol (100\%, 75\%, 50\% in water), and acetone (100\%, $75 \%$, and $50 \%$ in water). Results showed that the highest total flavonoids content was detected after extraction with 100\% ethanol solution. This finding suggested that flavonoids are soluble in organic solvents. However, flavonols content in plants may have different polarity depending on the attachment of sugar moiety. Therefore, a mixture of organic and polar solvents might enhance the extraction of plant-derived flavonols. 
In another study, flavonol glycosides in plums were extracted in $80 \%$ methanol aqueous solution [84]. Extracted flavonols were acid hydrolyzed to quantify total flavonols content in different vegetables. All desired flavonols were extracted and detected, suggesting that $80 \%$ aqueous methanol solution is enhanced the extraction of plant flavonols. This method was also used in another study to optimally extract flavonols from black and brown soybeans with the addition of 5\% formic acid [85].

The form of plant material is another important factor to consider for the extraction step. Increasing the surface area and reducing the particle size of the plant sample can be achieved by pulverization to expose plant tissues and content to the extraction solvents [86]. For this step, plant samples are usually freeze dried. It has been reported that non-thermally dried plants (lyophilization) result in higher flavonoids detection compared to thermal drying or fresh samples [87].

Extraction technique allows a non-selective separation of all plant compounds that dissolve in the extraction solutions. However, to selectively identify and detect molecules in plant extract, isolation technique is the second step in the identification of plant-derived flavonols.

\section{Isolation and Detection of Flavonol Glycosides}

Isolation is an important step, after extraction, to selectively detect desired compounds from other interfering plant materials in the plant extract [82]. Isolation techniques are usually applied to separate specific molecules depending on the nature of the desired compounds.

Chromatography is one of the most effective and selective isolation procedures that developed overtime [88]. Old chromatography technique includes simple thin layer chromatography (TLC), preparative thin layer chromatography (PTLC), and open column 
chromatography (CC). The mechanism of separation using chromatography is based on the polarity of the desired molecule [88]. For these techniques, clear plant extract and separation column are required. The separation column is a column or plate coated with a specific material that varies depending upon the polarity of the desired compounds [89]. Polar molecules are separated using polar columns such as columns coated with silica, which will be separated by running non-polar solvents through the column. This technique allows the desired polar compounds to bind to the column eliminating less polar molecules. The recent isolation methods include high-performance liquid chromatography (HPLC) and ultra-high-performance liquid chromatography (UHPLC).

The advanced methods are more accurate and require protocol development to determine specific conditions (column selection, flow rate, and mobile phase solvents) to accurately isolate the desired compounds from a particular plant [89]. The protocol varies depending on the type of column and plant used for analysis [90]. To optimally detect and quantify flavonols glycoside in ramps, a new UHPLC method needs to be developed for optimal separation and detection of flavonol glycosides. Table 3 shows different conditions for flavonols isolation.

Although the previously mentioned detection methods aid in the analysis of flavonol glycosides in plants, these methods are inaccurate for the characterization of unknown conjugates because it requires the availability of commercial standards to confirm the presence of particular conjugates. Due to the wide variations of flavonol glycosides synthesized in plants, some complex conjugates do not have commercial standards and need further characterization techniques to identify structures. 
Table 3. Characteristics of different chromatography conditions for the detection of flavonol glycosides.

\begin{tabular}{|c|c|c|c|c|c|}
\hline Plant & Flavonols detected & Mobile Phase & Column & Method & Ref \\
\hline $\begin{array}{l}\text { Onion, spinach, } \\
\text { orange and tomato }\end{array}$ & $\begin{array}{c}\text { Quercetin } \\
\text { 4'-O-glucoside \& } \\
\text { 3,4'di-O-glucoside }\end{array}$ & $\begin{array}{l}\text { Solvent A: methanol } \\
\text { Solvent B: ethyl } \\
\text { acetate }\end{array}$ & $\begin{array}{l}\text { A Luna C18 } \\
\text { reverse phase } \\
\text { column }(5 \mu \mathrm{m}, \\
250 \mathrm{~mm})\end{array}$ & HPLC & [91] \\
\hline Soybeans & $\begin{array}{l}\text { Kaempferol-3- } \\
\text { rhamnoglucoside }\end{array}$ & $\begin{array}{l}\text { Solvent A: } 0.1 \% \\
\text { formic acid in water } \\
\text { Solvent B: } 0.1 \% \\
\text { formic acid in } \\
\text { acetonitrile }\end{array}$ & $\begin{array}{c}\text { Symmetry C18 } \\
\text { column }(4.6 \mathrm{~mm} \\
\times 75 \mathrm{~mm}, 3.5 \\
\mu \mathrm{m})\end{array}$ & HPLC & {$[92]$} \\
\hline Plums & $\begin{array}{c}\text { Quercetin 3- } \\
\text { galactoside; quercetin } \\
\text { 3-glucoside }\end{array}$ & $\begin{array}{c}\text { Solvent A: } 50 \mathrm{mM} \\
\text { ammonium } \\
\text { phosphate } \\
\text { Solvent B: } \\
\text { acetonitrile:50 mM } \\
\text { of ammonium } \\
\text { phosphate } \\
\text { Solvent C: } 200 \mathrm{mM} \\
\text { phosphoric acid }\end{array}$ & $\begin{array}{l}\text { C18 reversed- } \\
\text { phase Symmetry } \\
\text { Analytical } \\
\text { column }(5 \mu \mathrm{m} \times \\
250 \mathrm{~mm} \times 4.6 \\
\mathrm{~mm})\end{array}$ & HPLC & [84] \\
\hline Onion & $\begin{array}{l}\text { Quercetin 3,4’- } \\
\text { diglucoside; } \\
\text { quercetin 4'- } \\
\text { monoglucoside }\end{array}$ & $\begin{array}{c}\text { Solvent A: } 0.1 \% \\
\text { formic acid in water } \\
\text { Solvent B: } 0.1 \% \\
\text { formic acid in } \\
\text { methanol }\end{array}$ & $\begin{array}{c}\text { Prevail C18 } \\
\text { column }(2.1 \times \\
150 \mathrm{~mm} 3 \mu \mathrm{m})\end{array}$ & HPLC & [93] \\
\hline Cranberry & $\begin{array}{l}\text { Quercetin: } \\
\text {-glucoside, } \\
\text {-galactoside, } \\
\text {-xylopyranoside } \\
\text { Kaempferol; } \\
\text {-methoxy } \\
\end{array}$ & $\begin{array}{l}\text { Solvent A: } 2 \% \\
\text { formic acid } \\
\text { Solvent B: } 2 \% \\
\text { formic acid in } \\
\text { methanol }\end{array}$ & $\begin{array}{l}\text { Zorbax SB-C18 } \\
\text { reversed phase } \\
\text { column }(250 \mathrm{~mm} \\
\text { x } 4.6 \mathrm{~mm})\end{array}$ & HPLC & [94] \\
\hline Blueberries & $\begin{array}{l}\text { Quercetin: 3- } \\
\text { rutinoside, } \\
\text { galactoside, } \\
\text { glucoside }\end{array}$ & $\begin{array}{c}\text { Solvent A: } 2 \% \\
\text { acetic acid in water } \\
\text { Solvent B: } 0.5 \% \\
\text { acetic acid in } \\
\text { acetonitrile/water } \\
(50: 50)\end{array}$ & $\begin{array}{l}\text { Aqua 3u, C18 } \\
(150 \times 2 \mathrm{~mm})\end{array}$ & HPLC & [95] \\
\hline
\end{tabular}




\section{Structural Characterization of Flavonol Glycosides}

Advancements in the detection methods of different metabolites enhanced the understanding of different plants bioactive compounds. Flavonoids gained more attention in both plant science and pharmaceutical fields after the development of specific characterization methods [96]. Characterization of flavonol glycosides can be achieved using the molecular mass of the desired compounds and the physical characteristics under magnetic fields.

Mass spectrometry uses the mass-to-charge ratio $(\mathrm{m} / \mathrm{z})$ to isolate and detect the compounds [97]. This technique requires the ionization of species by adding or subtracting an atom which is most commonly $\mathrm{H}, \mathrm{Cl}$, or $\mathrm{Na}$. The ionized molecules then undergo conversion to the gas phase for the detection [97]. After the detection of the desired flavonol glycosides under the specific wavelength of $350 \mathrm{~nm}$, resulting mass-to-charge ratio $(\mathrm{m} / \mathrm{z})$ will be used to characterize flavonol glycosides based on the molecular weight [98]. The molecular weight of quercetin and kaempferol backbones are 302 and $286 \mathrm{~g} / \mathrm{mol}$, respectively [99]. The attachment of different sugar moieties causes variation in the molecular weight of quercetin and kaempferol conjugates. However, mass spectrometry fails to provide information on the specific sugar moiety isomers attached to the flavonol backbone and the position of attachment. Therefore, a more advanced structure characterization method is required.

Nuclear magnetic resonance (NMR) spectroscopy is used for structural elucidation of complex conjugates by the detection of electromagnetic signals produced from particular atoms [100]. The magnetic resonance of carbon $\left({ }^{13} \mathrm{C}\right)$ and hydrogen $\left({ }^{1} \mathrm{H}\right)$ are used for NMR analysis [101]. This method is used to identify unknown complex flavonol glycosides that were not identified by mass spectrometry. Shahat et al., identified different unknown quercetin structures from methanolic extract of Carrichtera annual L. seeds [102]. Also, Kaempferol conjugate 
(kaempferol-3-O-hydroxyferuloylsophoroside-7-O-glucoside) from Chinese cabbage was characterized using NMR [103].

To analyze flavonol glycosides from ramps (Allium tricoccum), a separation protocol of the flavonol glycosides need to be developed. Additionally, analysis using UHPLC and mass spectrometry is required. If the detected flavonol glycosides are not available in commercial standards, NMR analysis is also needed to confirm the sugar moieties detected by mass spectrometry. 


\section{CHAPTER 3: METHODS}

\section{A. Separation and Characterization of Major Flavonol Glycosides}

\section{Chemicals}

Extraction solvents, including methanol and acetic acid were purchased from Fisher Scientific (USA). Hydrochloric acid, kaempferol (>90\% purity) and quercetin aglycone standards were purchased from Sigma Aldrich (USA). All solvents for ultra-high-performance liquid chromatography (UHPLC) and mass spectrometry (LC-MS/MS) were purchased from Fisher Scientific (USA).

\section{Plant Material}

Ramps were harvested from West Virginia. Ramps were cleaned and stored in a $-20^{\circ} \mathrm{C}$ freezer until used for analysis. Ramps samples were lyophilized and pulverized for analysis because it was reported that this technique maximizes flavonols extraction [87]. Whole ramps were lyophilized (BenchTop Pro, SP Scientific, PA, USA) for six days at $-46^{\circ} \mathrm{C}$. A portion of the lyophilized ramps were separated to bulb, stem, and leaves by cutting the middle purple stem from both ends. The second portion of lyophilized ramps was kept whole. The lyophilized individual parts and whole ramp samples were pulverized separately. The powder of each ramp part and whole ramps were packaged in a small transparent ziplock bags and stored at $-80{ }^{\circ} \mathrm{C}$.

\section{Extraction of Flavonol Glycosides}

For extraction of flavonol glycosides, each sample was prepared by homogenizaing $5 \mathrm{mg}$ of the pulverized whole ramps or individual parts in a $100 \mu \mathrm{L}$ of a solution containing $50 \%$ methanol and 3\% acetic acid in distilled water (v/v). The prepared sample was wrapped with aluminum foil to prevent exposure to light and mixed overnight ( 18 hours) at room temperature. Extracted samples were centrifuged for 3 minutes (13.3 g, ThermoScientific, CA, 
USA). The supernatant was transferred to micro-filters and centrifuged for other 3 minutes (13.3

g, ThermoScientific, CA, USA). The filtered solvent was diluted with $200 \mu \mathrm{L}$ of a solution containing $3 \%$ acetic acid in distilled water ( $\mathrm{v} / \mathrm{v})$ and the resulting solution was transferred to a UHPLC test vial and stored in $-80^{\circ} \mathrm{C}$ until it was used for analysis.

\section{Flavonol Glycoside Separation}

To identify flavonol glycosides in ramps, a separation protocol first had to be developed. UHPLC- photodiode array (PDA) was conducted using an Accela system (Thermo Scientific, San Jose, CA, USA) consisting of Accela 1250 pump, open autosampler, and PDA detector. Flavonol chromatographic separation was performed on a Luna Omega 1.6 $\mu$ m Polar C18 100Å, LC column $150 \times 2.5 \mathrm{~mm}$ (phenomenex, CA, USA) at $35^{\circ} \mathrm{C}$. The flow rates 300 and 500 $\mu \mathrm{L} /$ minute were tested. Gradient elution rates of a mobile phase for solvent A [ $5 \%$ formic acid in distilled water (v/v)], and solvent B [ $5 \%$ formic acid in acetonitrile (v/v)] were adjusted as shown in table 1. Mobile phase solvents were selected based on a previously reported method [85]. An injection volume of $5 \mu \mathrm{L}$ was held consistent throughout different methods. Flavonols were detected under a wavelength of $350 \mathrm{~nm}$. 
Table 1. Mobile phase gradient elution of different flow rates and gradient elution times.

\begin{tabular}{|c|c|c|}
\hline Method & Solvent A & Solvent B \\
\hline \multicolumn{3}{|c|}{ Flow rate $(\mu \mathrm{L} /$ minute $)$} \\
\hline 500 & $\begin{array}{c}0 \text { min, } 90 \% ; 2 \text { min, } 75 \% ; 7 \\
\text { min, } 65 \% \text {; } 9 \text { min, } 0 \% ; 11 \text { min, } \\
0 \% ; 12 \text { min, } 90 \% ; 15 \text { min, } \\
90 \%\end{array}$ & $\begin{array}{l}0 \mathrm{~min}, 10 \% ; 2 \mathrm{~min}, 25 \% ; 7 \mathrm{~min} \text {, } \\
\text { 35\%; } 9 \mathrm{~min}, 100 \% ; 11 \mathrm{~min}, \\
100 \% ; 12 \mathrm{~min}, 10 \% ; 15 \mathrm{~min}, 10 \%\end{array}$ \\
\hline \multicolumn{3}{|c|}{ Gradient elution time (minutes) } \\
\hline 15 & $\begin{array}{c}0 \text { min, } 90 \% ; 2 \text { min, } 75 \% ; 7 \\
\text { min, } 65 \% \text {; } 9 \text { min, 0\%; } 11 \text { min, } \\
0 \% ; 12 \text { min, } 90 \% ; 15 \text { min, } \\
90 \%\end{array}$ & $\begin{array}{l}0 \mathrm{~min}, 10 \% \text {; } 2 \mathrm{~min}, 25 \% ; 7 \mathrm{~min} \text {, } \\
\text { 35\%; } 9 \mathrm{~min}, 100 \% ; 11 \mathrm{~min}, \\
100 \% ; 12 \mathrm{~min}, 10 \% ; 15 \mathrm{~min}, 10 \%\end{array}$ \\
\hline 21 & $\begin{array}{c}0 \min , 90 \% ; 6 \min , 90 \% ; 8 \\
\min , 75 \% ; 13 \min , 65 \% ; 15 \\
\min , 0 \% ; 17 \min , 0 \% ; 18 \min \text {, } \\
90 \% ; 21 \min , 90 \%\end{array}$ & $\begin{array}{c}0 \mathrm{~min}, 10 \% \text {; } 6 \min , 10 \% \text {; } 8 \mathrm{~min} \text {, } \\
\text { 25\%; } 13 \mathrm{~min}, 35 \% ; 15 \mathrm{~min} \text {, } \\
100 \% \text {; } 17 \mathrm{~min}, 100 \% ; 18 \mathrm{~min} \text {, } \\
10 \% ; 21 \mathrm{~min}, 10 \%\end{array}$ \\
\hline 24 & $\begin{array}{c}0 \min , 98 \% ; 2 \min , 98 \% ; 3 \\
\min , 90 \% ; 9 \min , 90 \% ; 11 \\
\min , 75 \% ; 16 \min , 65 \% ; 18 \\
\min , 0 \% ; 20 \min , 0 \% ; 21 \min \text {, } \\
\text { 98\%; } 24 \min , 98 \%\end{array}$ & $\begin{array}{c}0 \text { min, } 2 \% ; 2 \min , 2 \% ; 3 \min \text {, } \\
10 \% \text {; } 9 \text { min, } 10 \% ; 11 \text { min, } 25 \% \text {; } \\
16 \text { min, } 35 \% \text {; } 18 \text { min, } 100 \% ; 20 \\
\min , 100 \% ; 21 \min , 2 \% ; 24 \min \text {, } \\
2 \%\end{array}$ \\
\hline
\end{tabular}

\section{Characterization of Flavonol Glycosides by UHPLC-PDA-MS ${ }^{2}$}

To characterize flavonol glycosides, UHPLC-PDA-MS² was conducted using an Accela system (Thermo Scientific, San Jose, CA, USA) consisting of Accela 1250 pump, open auto sampler, and PDA detector connected to a Q-Exactive-Oribitrap MS containing a heated electrospray ionization (HESI). Flavonols chromatographic separation was performed on a Luna Omega $1.6 \mu$ m Polar C18 100Å, LC column 150 X 2.5 mm (phenomenex, CA, USA) at $35^{\circ} \mathrm{C}$. The optimal chromatographic conditions based on the UHPLC protocol development results were gradient elution with a mobile phase, solvent A; 5\% formic acid in LC-MS grade water (v/v), solvent B; 5\% formic acid in acetonitrile (v/v). The gradient was programmed as follows: 0 min, 2\% B; 3 min, 10\% B; 11 min, 25\% B; 16 min, 35\% B; 18 min, 100\% B; 21 min, 2\% B; $24 \mathrm{~min}, 0 \% \mathrm{~B}$. For column wash, solvent C; 60\% acetonitrile was used for 10 minutes. The flow rate was $300 \mu \mathrm{L} /$ min throughout the gradient, with an injection volume of $5 \mu \mathrm{L}$. 
MS analysis was performed in Full MS in both positive and negative polarity modes. More efficient detection was observed under the negative mode. Thus, all analysis was conducted under negative polarity mode. Full MS properties were: resolution 70,000, AGC target 3e6, maximum IT $100 \mathrm{~ms}$, and scan range 200-2000 m/z. Compounds were identified by analyzing parent to fragments, and data dependent fragmentation was conducted to detect the fragments of the major parent peaks. Tentative conjugates were identified by comparing the detected $\mathrm{m} / \mathrm{z}$ values within $10 \mathrm{ppm}$ to similar values using Metabolite and Chemical Entity Database (METLIN) and published data. The properties of data dependent fragmentation were; resolution 35,000, AGC target 2e5, maximum IT 50 ms, loop count 5, (N)CE 33, charge exclusion unassigned 3-8, >8.

Flavonols were detected at $350 \mathrm{~nm}$ wavelength. All chromatogram data were collected and analyzed using Xcalibur software (Thermo Scientific, San Jose, CA, USA). HESI source properties were; nitrogen gas with sheath gas flow rate 18, Aux gas flow rate 11, spray voltage (kV) 2.5, capillary temperature $320^{\circ} \mathrm{C}$. Potential chemical structures detected by UHPLC-PDAMS $^{2}$ based on $m / z$ values reported in the literature, were constructed in ChemDraw Prime 16.0.

\section{Preliminary NMR Analysis}

To confirm the tentatively characterized structures, a preliminary nuclear magnetic resonance (NMR) experiment was conducted using the flavonol glycosides extract. The prepared extract was dried under gas nitrogen, the last drying step was achieved by lyophilizing the concentrated sample. For NMR measurements, 20-30mg of the dried extraxt was dissolved in 0.7 mL DMSO-d6. The ${ }^{1} \mathrm{H}$ and ${ }^{13} \mathrm{C}$ NMR spectra were acquired at $25^{\circ} \mathrm{C}$ on a Varian INOVAUnity $600 \mathrm{MHz}$ equipped with a $5 \mathrm{~mm}$ inverse broadband PFG Probe. The FIDs of the 1H and 13C NMR spectra were processed using the commercially available NMR software 
package ACD/Spectrus Processor (version: S80S41, Build 108238, 08 Apr 2019, www.acdlabs.com ). ${ }^{1} \mathrm{H}$ NMR chemical shifts were reported relative to the internal residual solvent peaks at $2.5 \mathrm{ppm}$ (DMSO-d6). ${ }^{13} \mathrm{C}$ NMR chemical shifts were reported relative to the central peak of the septet was set to $39.51 \mathrm{ppm}$.

After the characterization of the major flavonol glycosides in whole ramps and individual parts, analysis of the total flavonol content is required. To quantify total flavonol in ramps, sugar moieties attached to the flavonol backbone need to be hydrolyzed. Due to the absence of previous reports on the total flavonol content in ramps, a preliminary development of hydrolysis protocol was conducted.

\section{B. Preliminary Development of Acid Hydrolysis Protocol for Quantification of Flavonol}

\section{Aglycones.}

Preparation of Aglycone Standard Solutions

UHPLC-grade quercetin and kaempferol aglycone standards were used. Kaempferol standard stock solution with a concentration of $100 \mu \mathrm{M}$ was prepared by dissolving the standard $(0.3 \mathrm{mg})$ in a $10 \mathrm{ml}$ solution of $50 \%$ methanol and water and sonicated for 2 minutes. Quercetin stock solution was prepared by diluting $10 \mathrm{mM}$ stock solution to $100 \mu \mathrm{M}$ in $50 \%$ methanol. Quercetin and kaempferol standards were used to detect hydrolyzed aglycones.

Development of Acid Hydrolysis Protocol

To quantify flavonol aglycones in ramps, the flavonol glycosides were subjected to acid hydrolysis. Different hydrolysis factors were examined, including hydrolysis time, starting material (plant tissues versus flavonol extract), and acid concentration. To test hydrolysis time, the hydrolysis method conducted by Nuutila et al. was modified [23]. To prepare samples, $2 \mathrm{ml}$ of $1.2 \mathrm{M} \mathrm{HCl}$ in $50 \%$ methanol solution was used to hydrolyze $20 \mathrm{mg}$ of pulverized whole 
ramps. Five samples (250 $\mu \mathrm{l}$ each) were hydrolyzed in a water bath at $80^{\circ} \mathrm{C}$ for $2,4,6,8$, and 12 hours. After hydrolysis, $250 \mu \mathrm{l}$ distilled water was added to each sample. Diluted samples were sonicated for 2 minutes, centrifuged for 2 minutes at speed $13.3 \mathrm{~g}$ (ThermoScientific, CA, USA). The resulting supernatant was filtered in a microfilter tube by transferring the supernatant to the filter and centrifuged for 3 minutes at speed $13.3 \mathrm{~g}$. The hydrolyzed solution was transferred to UHPLC test vials and placed in $-80^{\circ} \mathrm{C}$ until analysis.

Several hydrolysis studies performed flavonol glycoside hydrolysis using either plant tissues or flavonol glycoside extracts $[23,84,104]$. Therefore, the type of hydrolysis starting material was investigated by direct hydrolysis of pulverized whole ramps or hydrolysis of whole ramp flavonol extracts. Direct whole ramp hydrolysis sample was prepared following the previous method used for the analysis of different time intervals at $80^{\circ} \mathrm{C}$ for 2 hours. Hydrolysis of plant extract was prepared based on the method reported [92,105]. A $200 \mu \mathrm{l}$ flavonol extract was previously prepared was hydrolyzed by adding $50 \mu \mathrm{l}$ of $1 \mathrm{M} \mathrm{HCl}$ and then placed in a water bath at $90^{\circ} \mathrm{C}$ for 2 hours. Samples from both methods were stored at $-80^{\circ} \mathrm{C}$ until analysis.

Based on results from the type of hydrolyzed sample, the effect of different acid concentration was examined by hydrolyzing $1.2 \mathrm{ml}$ flavonols extract. Samples were hydrolyzed by adding $300 \mu \mathrm{l}$ of $1,2,3,4,5$, and $6 \mathrm{M} \mathrm{HCl}$ and placed in a water bath at $90^{\circ} \mathrm{C}$ for 2 hours. Hydrolyzed samples were cooled at room temperature, transferred to UHPLC test vials and stored at $-80^{\circ} \mathrm{C}$ until analysis.

Analysis of the hydrolyzed quercetin and kaempferol aglycones was carried out on UHPLC system (Thermo Scientific, San Jose, CA, USA) consisting of Accela 1250 pump, open auto sampler, and PDA detector. An aliquot of $5 \mu \mathrm{L}$ was injected to the UHPLC system. The chromatographic separation was performed on a Luna Omega 1.6 $\mu$ m Polar C18 100A, LC 
column 150 X $2.5 \mathrm{~mm}$ (phenomenex, CA, USA) at $35^{\circ} \mathrm{C}$. The mobile phase solvents and chromatographic conditions were similar to conditions described above for UHPLC-PDA-MS except for the gradient elution rate. The gradient was programmed as follows: $0 \mathrm{~min}, 2 \% \mathrm{~B} ; 3$ min, 10\% B; 11 min, 25\% B; 16 min, 35\% B; 18 min, 100\% B; 21 min, 2\% B; 24 min, 0\% B. Flavonols were detected at wavelength of $350 \mathrm{~nm}$. All chromatogram data were collected and analyzed using Xcalibur software (Thermo Scientific, San Jose, CA, USA). 


\section{CHAPTER 4: RESULTS}

\section{A. Separation and Characterization of Major Flavanol Glycosides}

\section{Separation of Flavonol Glycosides}

Figure 1 shows the chromatogram results of different UHPLC methods at $350 \mathrm{~nm}$, including flow rate and gradient elution time. Peaks separation was not improved by increasing the flow rate from 300 to $500 \mu \mathrm{L} /$ minute for 15 minutes gradient elution time because it resulted in a faster elution of all peaks within a smaller range of time. Also, the peak at $\mathrm{R}_{\mathrm{t}} 2.34$ min in figure $1 \mathrm{~A}$ which eluted at $\mathrm{R}_{\mathrm{t}} 1.57$ in figure $1 \mathrm{~B}$ overlapped with other shoulder peak under both flow rates. Therefore to achieve a complete separation of peaks, a flow rate of $300 \mu \mathrm{L} / \mathrm{minute}$ will be used to examine the elution gradient time factor.

An increased gradient elution time was achieved by increasing the elution gradient of 90\% polar solvent A. This improved the separation of peaks. The elution time of 21 minutes resulted in the more complete separation and slower elution of the major peaks compared to the elution time of 15 minutes. However, the two peaks at $\mathrm{R}_{\mathrm{t}} 2.59$ min were not completely separated. An increase in the elution gradient of polar solvent A to $98 \%$ with longer elution time to a total of 24 minutes resulted in complete separation of all major peaks and increased the retention time $\left(\mathrm{R}_{\mathrm{t}}\right)$ of the detected compounds. To identify flavonol glycosides in ramps, the optimal separation method to be used for UHPLC and LC-MS is flow rate of $300 \mu \mathrm{L} / \mathrm{minute}$ and elution gradient time of 24 minutes ( 0 min, 98\%; 2 min, 98\%; 3 min, 90\%; 9 min, 90\%; 11 min, 75\%; 16 min, 65\%; 18 min, 0\%; 20 min, 0\%; 21 min, 98\%; 24 min, 98\%). 
A.

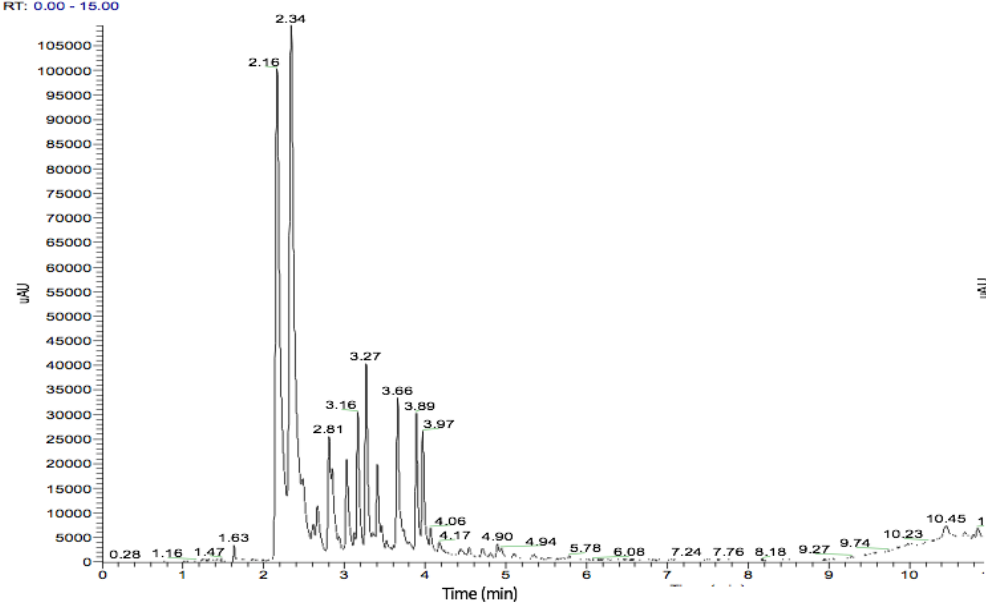

C. RT: $0.00-21.00$

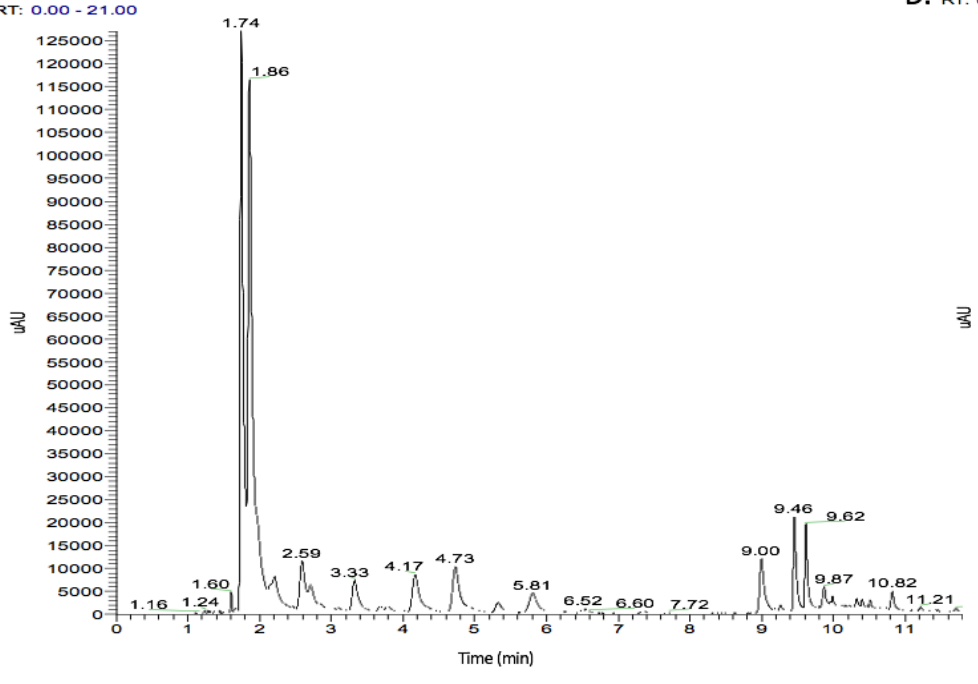

Figure 1. Chromatogram results of different UHPLC-PDA methods A) flow rate $300 \mu \mathrm{L} / \mathrm{minute}$ for 15 minutes, B) flow rate $500 \mu \mathrm{L} /$ minute for 15 minutes, C) 21 minutes gradient elution time with flow rate $300 \mu \mathrm{L} /$ minute, D) 24 minutes gradient elution time with flow rate $300 \mu \mathrm{L} /$ minute.
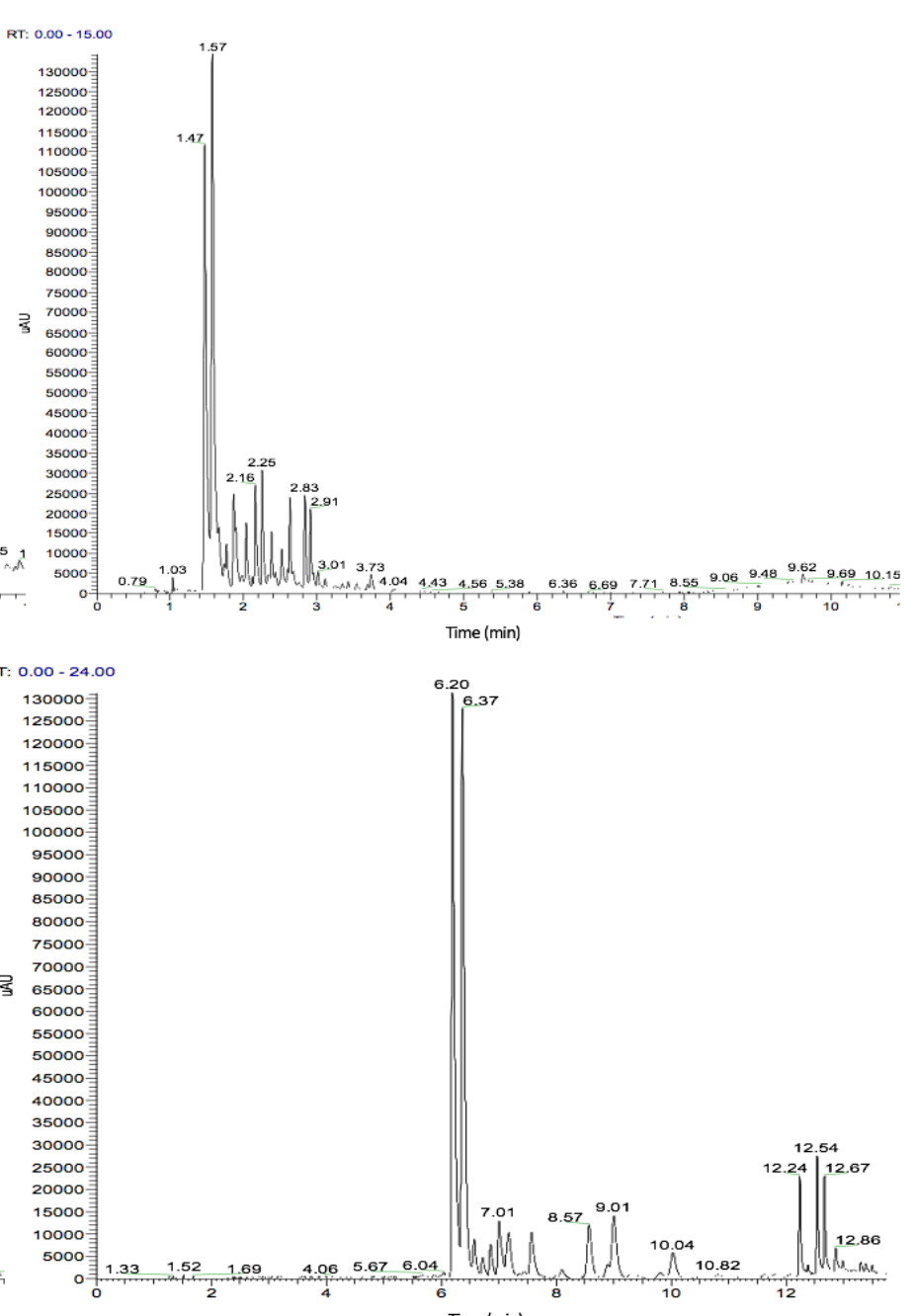

\section{.}




\section{Characterization of Flavonol Glycosides}

Table 2 shows flavonol glycosides that were putatively identified in whole ramps by UHPLC-PDA-MS² method along with with retention time $\left(\mathrm{R}_{\mathrm{t}}\right)$, putative chemical formula, [M$\mathrm{H}]^{-}(\mathrm{m} / \mathrm{z})$ values and the molecular weight. The major flavonol conjugates detected in whole ramps were quercetin and kaempferol glycosides. The first major peak corresponding to quercetin was detected at $\mathrm{R}_{\mathrm{t}} 6.63 \mathrm{~min}$, parent [M-H]- was $801.1719 \mathrm{~m} / \mathrm{z}$. Figure 2 shows the MS chromatogram of the parent masses and fragments of the major flavonol glycosides detected. Although chemical structures are shown, conjugates attachment positions can not be determined by LC-MS. Fragment of $301.0352 \mathrm{~m} / \mathrm{z}$ indicated the presence of quercetin backbone after the loss of linked sugar moieties. Also, fragment of $463.0879 \mathrm{~m} / \mathrm{z}$ indicates quercetin backbone with one hexoside attached, and fragment $625.1405 \mathrm{~m} / \mathrm{z}$ represents dihexoside. Based on METLIN database and data reported in litereature, the parent and fragments showed that the tentative structure of quercetin conjugate was quercetin sophoroside glucuronide [106]. A fragment corresponding to quercetin glucuronide connected to one hexoside was not detected indicating that the dihexoside attached was sohphoroside.

The second major peak eluted was detected at $\mathrm{R}_{\mathrm{t}} 6.77 \mathrm{~min}$, parent $[\mathrm{M}-\mathrm{H}]^{-}$was 785.1769 $\mathrm{m} / \mathrm{z}$ and fragments were $285.0246 \mathrm{~m} / \mathrm{z}$ which reflects kaempferol backbone after the fragmentation of the glycoside conjugates. Fragment of $446.0851 \mathrm{~m} / \mathrm{z}$ corresponding to kaempferol with one hexoside attached after the cleavage of other sugar moieties, and 609.1459 $\mathrm{m} / \mathrm{z}$. A fragment corresponding to kaempferol glucuronide connected to one hexoside was not detected indicating that the dihexoside attached was sohphoroside. Based on similar parent $\mathrm{m} / \mathrm{z}$ values, and some fragments reported in the literature, the tentative kaempferol structure is kaempferol sophoroside glucuronide [106]. A parent value of [M-H] $625.1398 \mathrm{~m} / \mathrm{z}$ at $\mathrm{R}_{\mathrm{t}} 12.65$ 
min with fragments at $301.0352 \mathrm{~m} / \mathrm{z}$ indicating quercetin backbone and $463.0890 \mathrm{~m} / \mathrm{z}$ representing quercetin attached to one hexoside after the fragmentation of one hexoside. The tentative structure of the second quercetin conjugate was identified, based on previous reports and METLIN database, as quercetin dihexoside [107,108].

Table 2. UHPLC-PDA-MS data and putative identification of flavonol glycosides in whole ramps.

\begin{tabular}{|c|c|c|c|c|c|c|}
\hline$\underset{\text { (minute) }}{\mathbf{R} \mathbf{t}}$ & $\begin{array}{c}{[\mathrm{M}-\mathrm{H}]^{-}} \\
(\mathrm{m} / \mathrm{z})\end{array}$ & $\begin{array}{l}\text { MS/MS } \\
(\mathrm{m} / \mathbf{z})\end{array}$ & $\begin{array}{c}\text { Molecular } \\
\text { weight } \\
\text { (g/mol) }\end{array}$ & $\begin{array}{l}\text { Molecular } \\
\text { formula }\end{array}$ & $\begin{array}{l}\text { Putative } \\
\text { compound }\end{array}$ & Ref $^{*}$ \\
\hline 6.63 & 801.1719 & $\begin{array}{l}301.0352, \\
625.1405, \\
463.0879\end{array}$ & 802 & $\mathrm{C}_{33} \mathrm{H}_{38} \mathrm{O}_{23}$ & $\begin{array}{l}\text { Quercetin } \\
\text { sophoroside } \\
\text { glucuronide }\end{array}$ & [106] \\
\hline 6.77 & 785.1769 & $\begin{array}{l}\text { 285.0246, } \\
446.0851, \\
609.1459\end{array}$ & 786 & $\mathrm{C}_{33} \mathrm{H}_{38} \mathrm{O}_{22}$ & $\begin{array}{l}\text { Kaempferol } \\
\text { sophoroside } \\
\text { glucuronide }\end{array}$ & [106] \\
\hline 12.65 & 625.1398 & $\begin{array}{l}\text { 301.0352, } \\
463.0890\end{array}$ & 626 & $\mathrm{C}_{27} \mathrm{H}_{30} \mathrm{O}_{17}$ & $\begin{array}{l}\text { Quercetin- } \\
\text { dihexoside }\end{array}$ & {$[107,108]$} \\
\hline
\end{tabular}

*all conjugates found in Metabolite and Chemical Entity Database (METLIN) 
A.
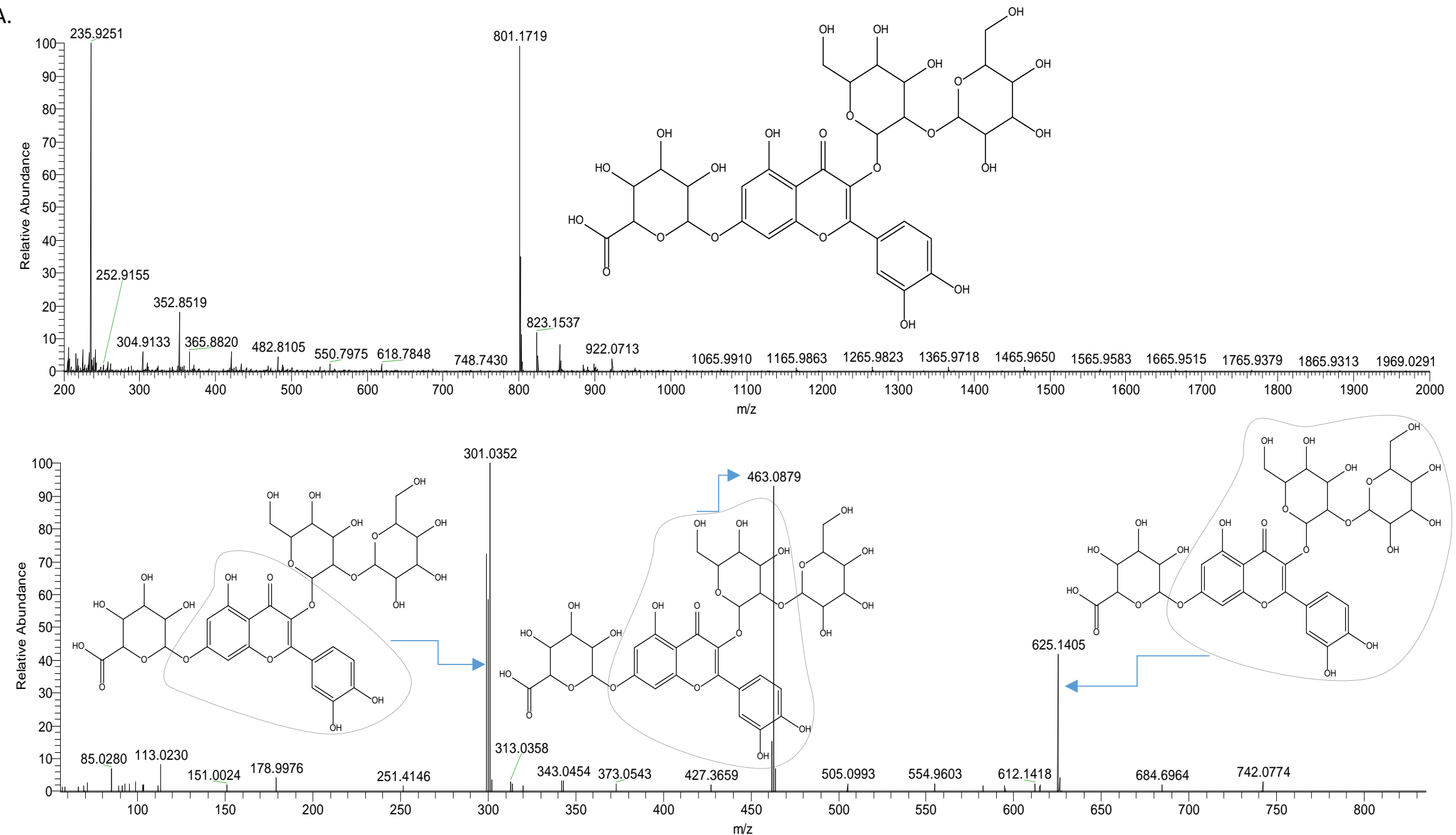
B.

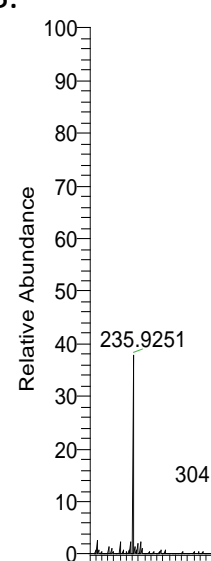

785.1769

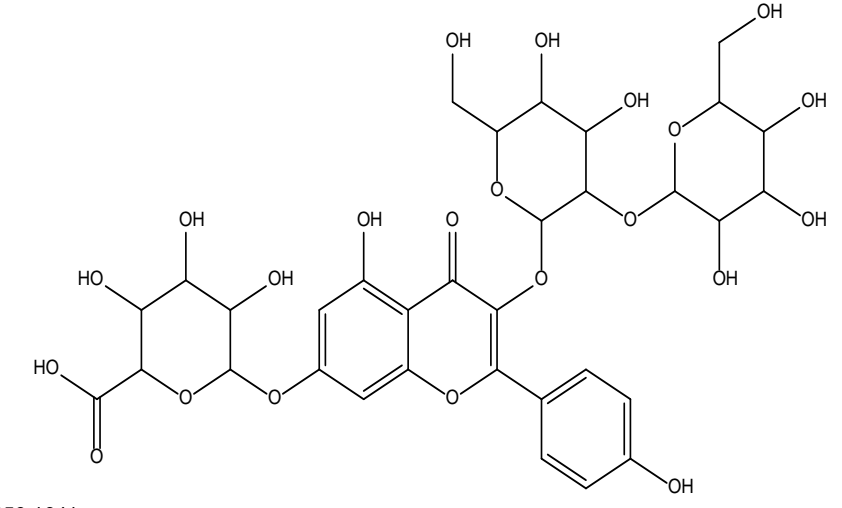

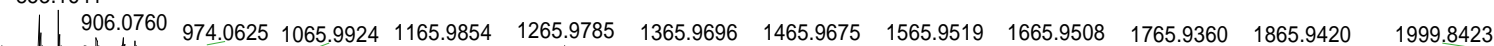

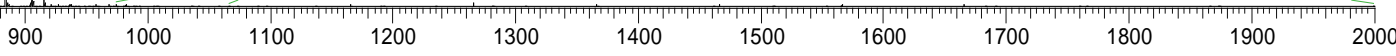
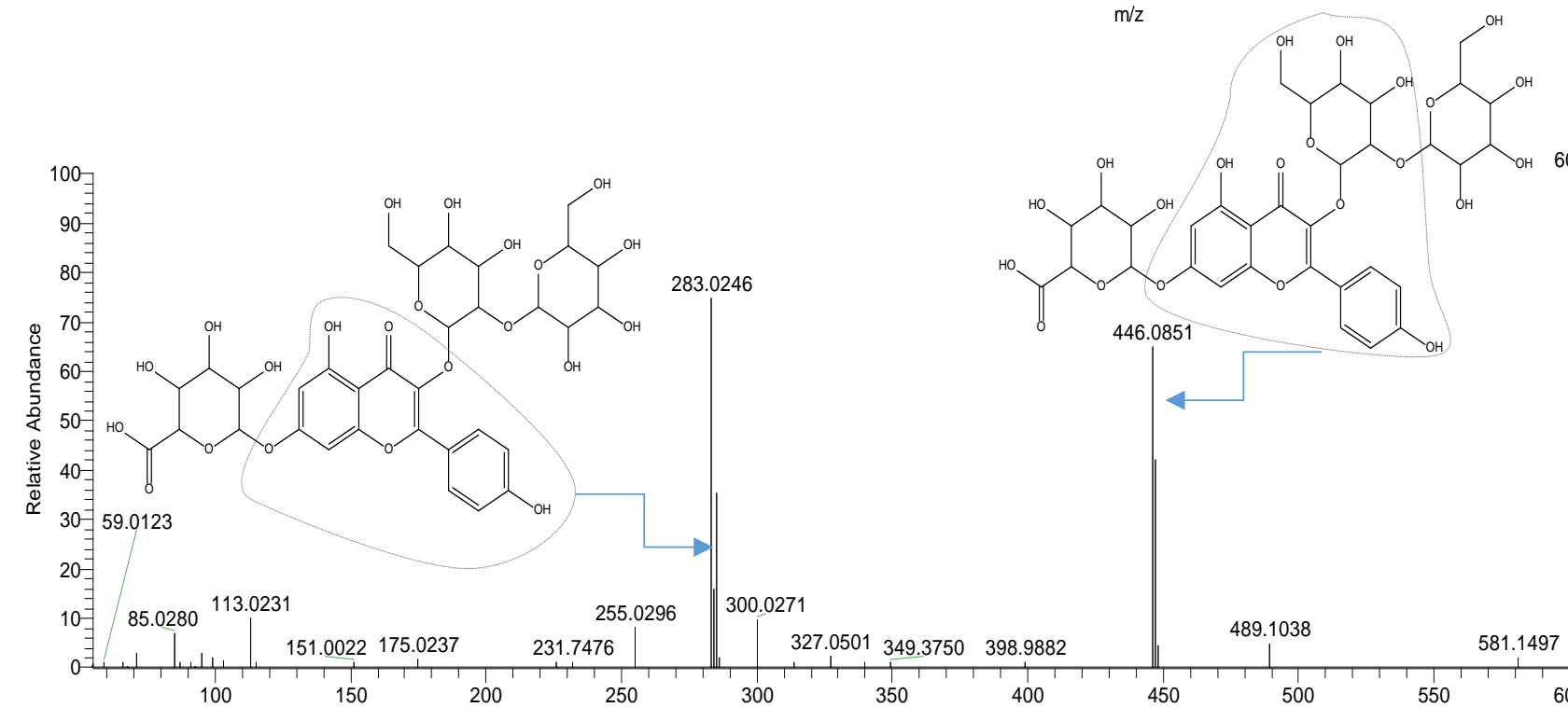

446.08
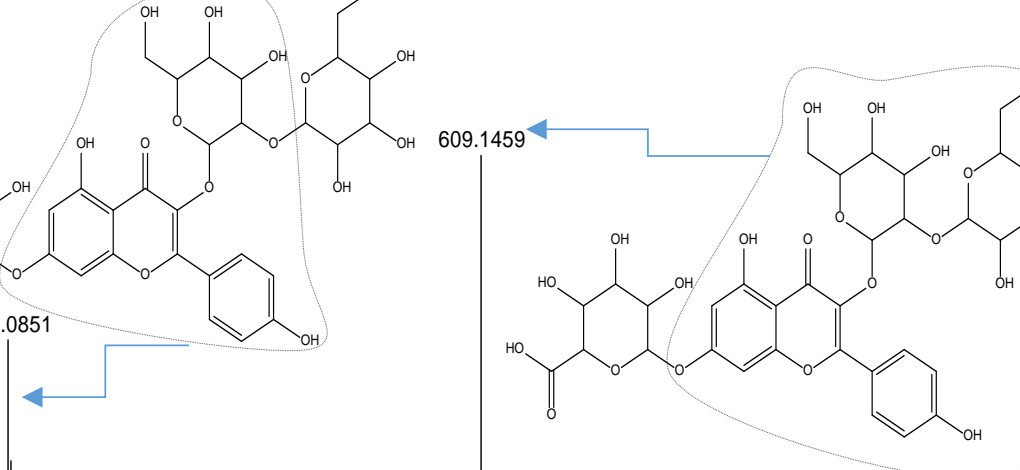

581.1497

713.2915741 .9855 

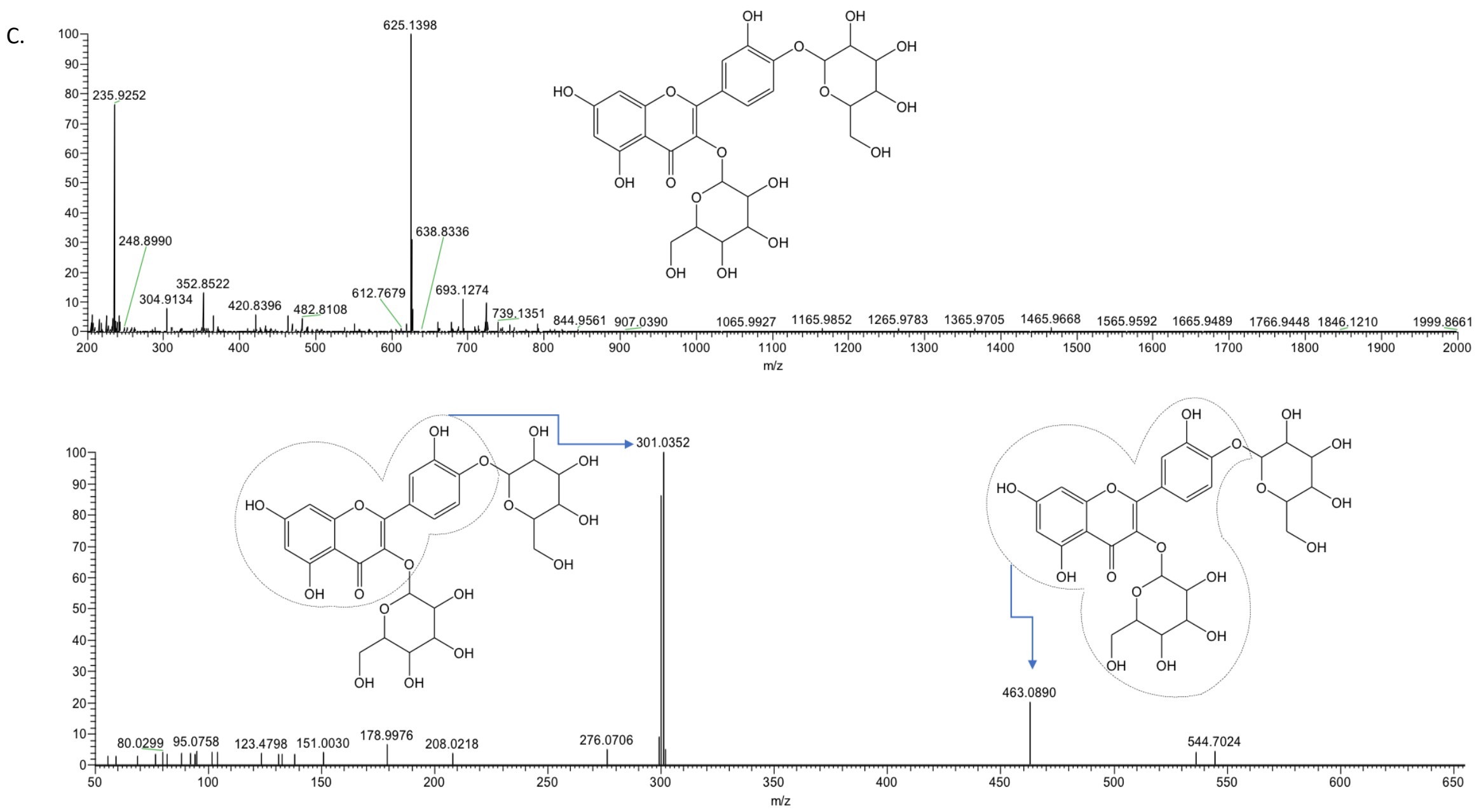

Figure 2. Representation of chromatogram data of negative mode MS (top) and data dependent fragmentation (bottom) of A) Quercetin sophoroside glucuronide, B) Kaempferol sophoroside glucuronide, C) Quercetin-dihexoside. Chemical structures do not represent conjugates attachment positions. 
Analysis of the major flavonol glycosides by UHPLC-PDA-MS ${ }^{\mathrm{n}}$ in individual parts showed that kaempferol sophoroside glucuronide, quercetin sophoroside glucuronide, and quercetin-dihexoside were present in the leaves and whole ramp. In the stem, only quercetin sophoroside glucuronide was detected. No peaks corresponding to either quercetin or kaempferol conjugates were detected in the bulb. Figure 3 represents the identification and relative intensities of major flavonol glycosides in whole ramps and individual parts. The intensity of the detected peaks by UHPLC-PDA was used to assess the relative concentration of the flavonol glycosides in individual parts. The majority of the flavonol glycosides were concentrated in the ramps' leaves. Kaempferol sophoroside glucuronide was higher than both quercetin sophoroside glucuronide and quercetin-dihexoside in both whole ramps and the leaves. The concentration of the detected flavonol glycosides in leaves was higher than individual parts and whole ramps. In the stem, trace concentration of quercetin sophoroside glucuronide was detected.

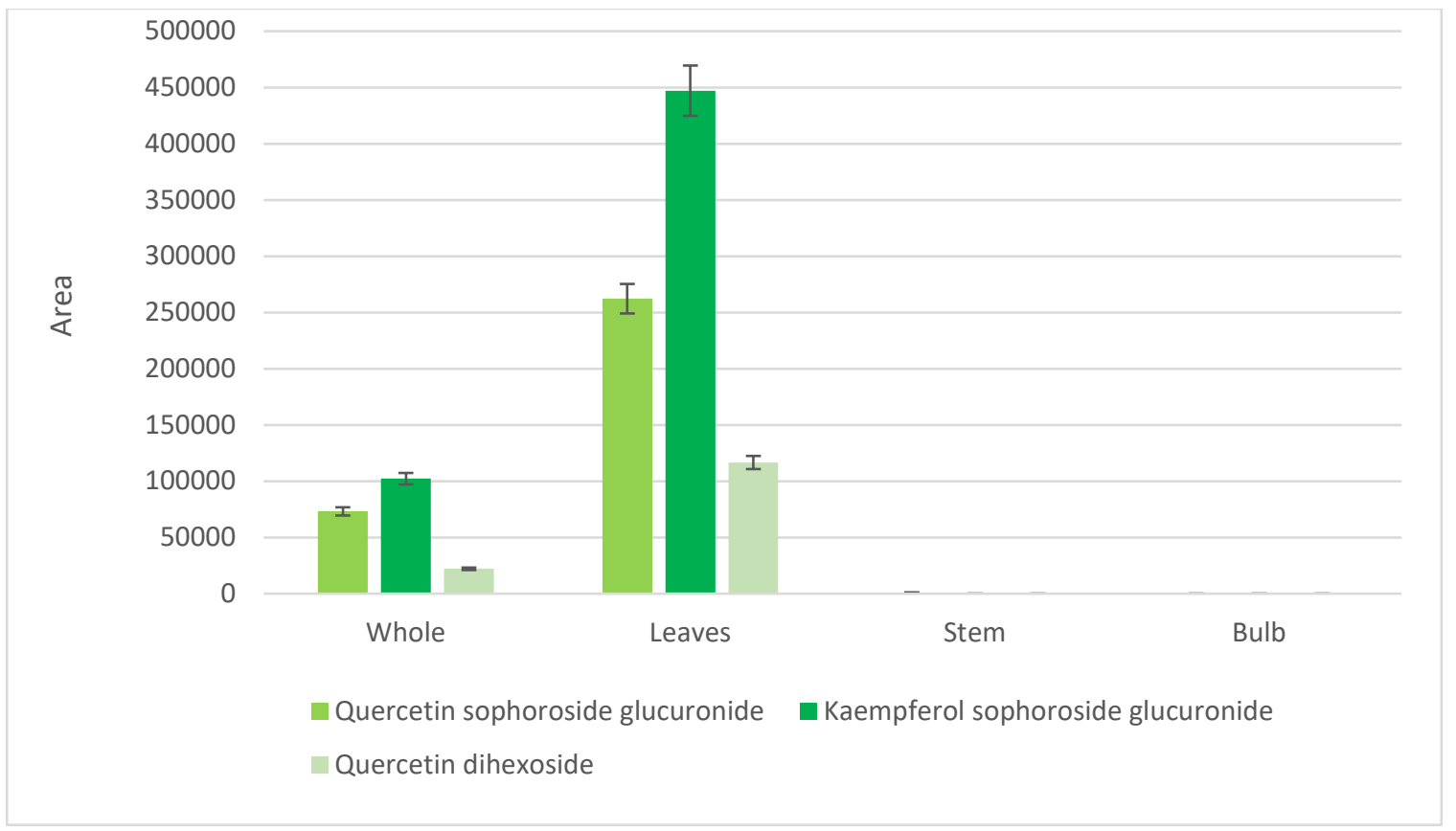

Figure 3. Characterization of the major flavonol glycosides detected in individual parts showing the intensity of the glycosides using area under the peak. 


\section{${ }^{1} \mathrm{H}$ and ${ }^{13} \mathrm{C}$ NMR Preliminary Structural Confirmation}

Figure 4 shows the ${ }^{1} \mathrm{H}$ NMR spectrum of the sugar moietis in the extract. The chemical shift between 3-5.8 ppm represent signals corresponding to the dihexoside, sophorosides, and monohexosides in the ramp extract. Based on signals detected in the aromatic region of 6.5-8.0 ppm, the presence of the aromatic rings that make up quercetin and kaempferol backbones confirmed at low intensity. Due to the low intensity of the the aromatic rings' proton signals, the spectrum was expanded to view the multiplicity pattern of the protons for further confirmation. Figure 5 shows the expanded ${ }^{1} \mathrm{H}$ NMR spectrum and expected multiplicity of the protons of quercetin and kaempferol backbone. Signals detected $\delta$ 6.82-6.87 and $\delta 6.42-6.44$ represent the protons on the aromatic rings of quercetin and kaempferol.

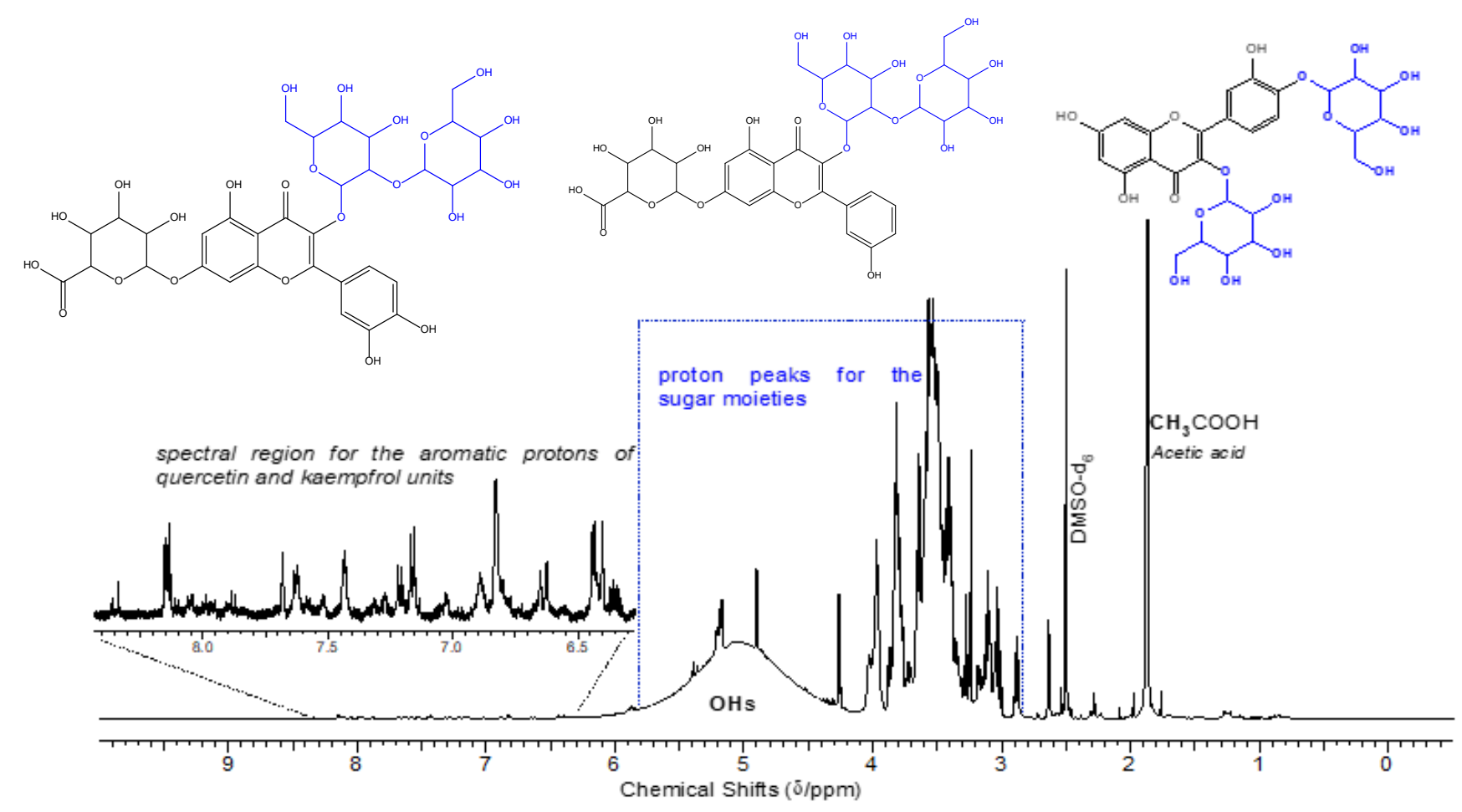

Figure 4. ${ }^{1} \mathrm{H}$ NMR spectrum of flavonol glycosides in DMSO-d6; the assignment of the individual proton resonances are not provided. 


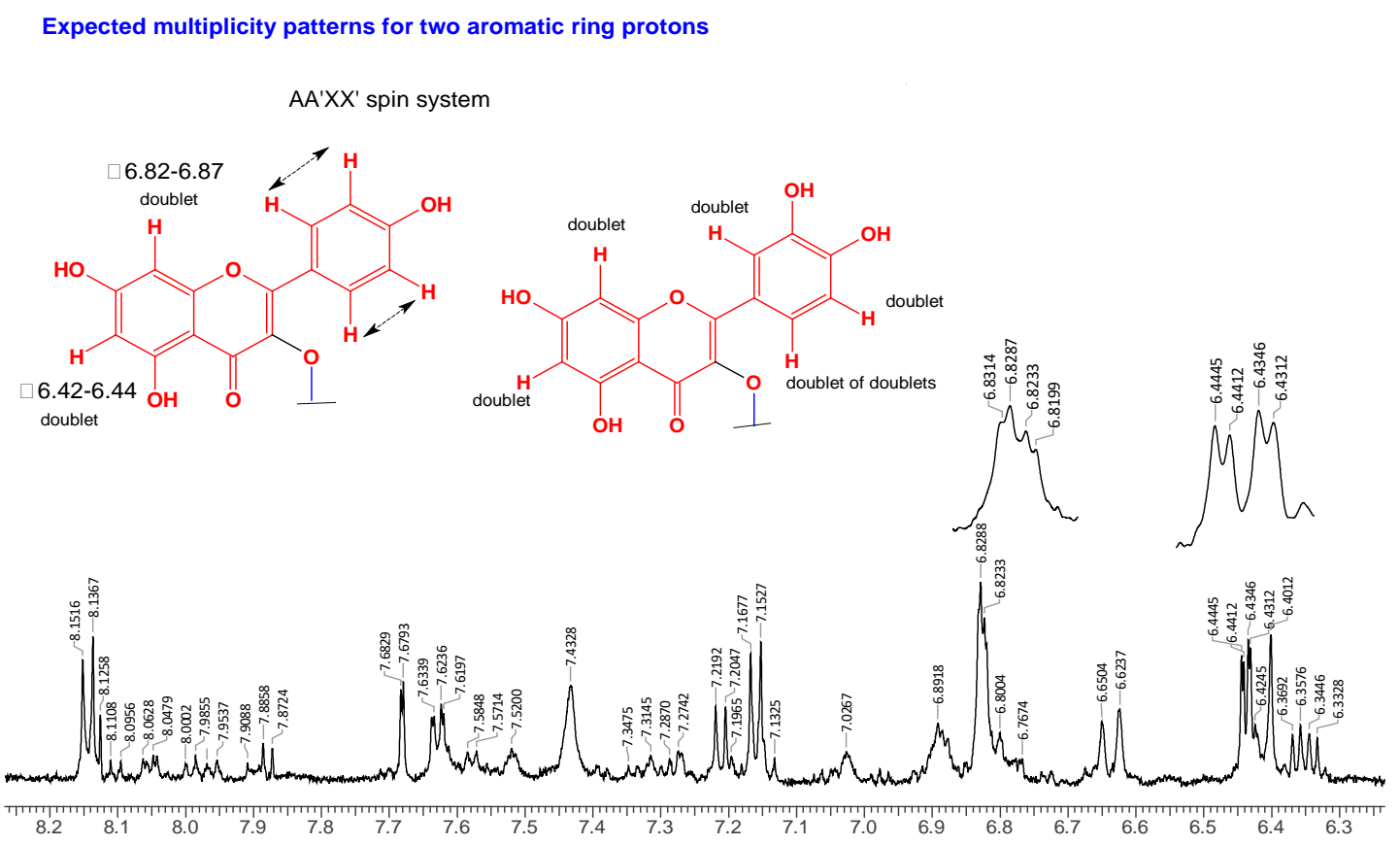

Figure 5. Expanded 1H NMR spectral region for the aromatic rings; expected multiplicity patterns (doublets and doublets of doublets) are clearly distinguishable in this region.

${ }^{13} \mathrm{C}$ NMR was conducted to detect signals corresponding to quercetin and kaempferol backbones. Figure 6 shows the chemical shifts of the carbon atoms corresponding to quercetin and kaempferol structure. Chemical shift between 60 and 65 ppm indicated the anomeric carbons $\left(-\mathrm{CH}_{3} \mathrm{OH}\right)$ on the flavonol backbone. The ${ }^{13} \mathrm{C}$ NMR spectrum confirmed the existence of quercetin and kaempferol backbones. 


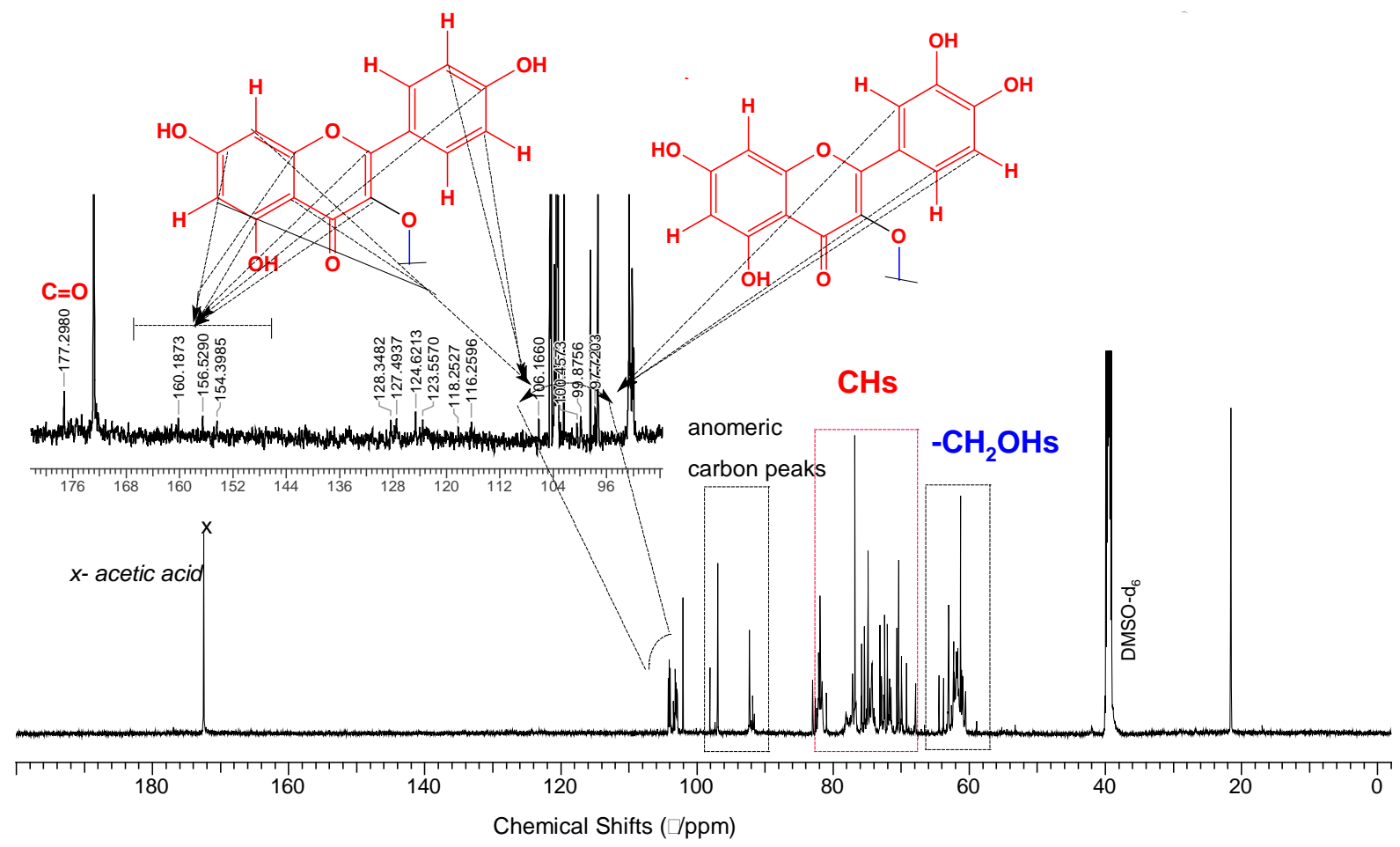

Figure 6. ${ }^{13} \mathrm{C}$ NMR spectrum of flavonol backbones in DMSO- $\mathrm{d}_{6}$; assignment of the individual carbon resonances are not given. $\mathrm{X}$; acetic acid from the extract. 


\section{B. Preliminary Development of Acid Hydrolysis Protocol for Quantification of Flavonol}

\section{Aglycones}

Figure 7 shows the retention time of $100 \mu \mathrm{M}$ quercetin and kaempferol aglycone standards. The $\mathrm{R}_{\mathrm{t}}$ of the standards will be used to detect hydrolyzed quercetin and kaempferol from whole ramps. Figure 8 represents the chromatogram results of the hydrolysis of whole ramps by $1.2 \mathrm{M} \mathrm{HCl}$ under different hydrolysis time intervals. The chromatogram results of different time intervals was compared with the retention time of quercetin and kaempferol standards (shown in figure 2). No hydrolysis of quercetin or kaempferol conjugates were detected. None of the hydrolyzed samples under different time intervals 2, 4, 6, 8, and 12 hours resulted in the detection of quercetin or kaempferol aglycones at or close to the $\mathrm{R}_{\mathrm{t}}$ of $7.11 \mathrm{~min}$ and 9.24 min, respectively.
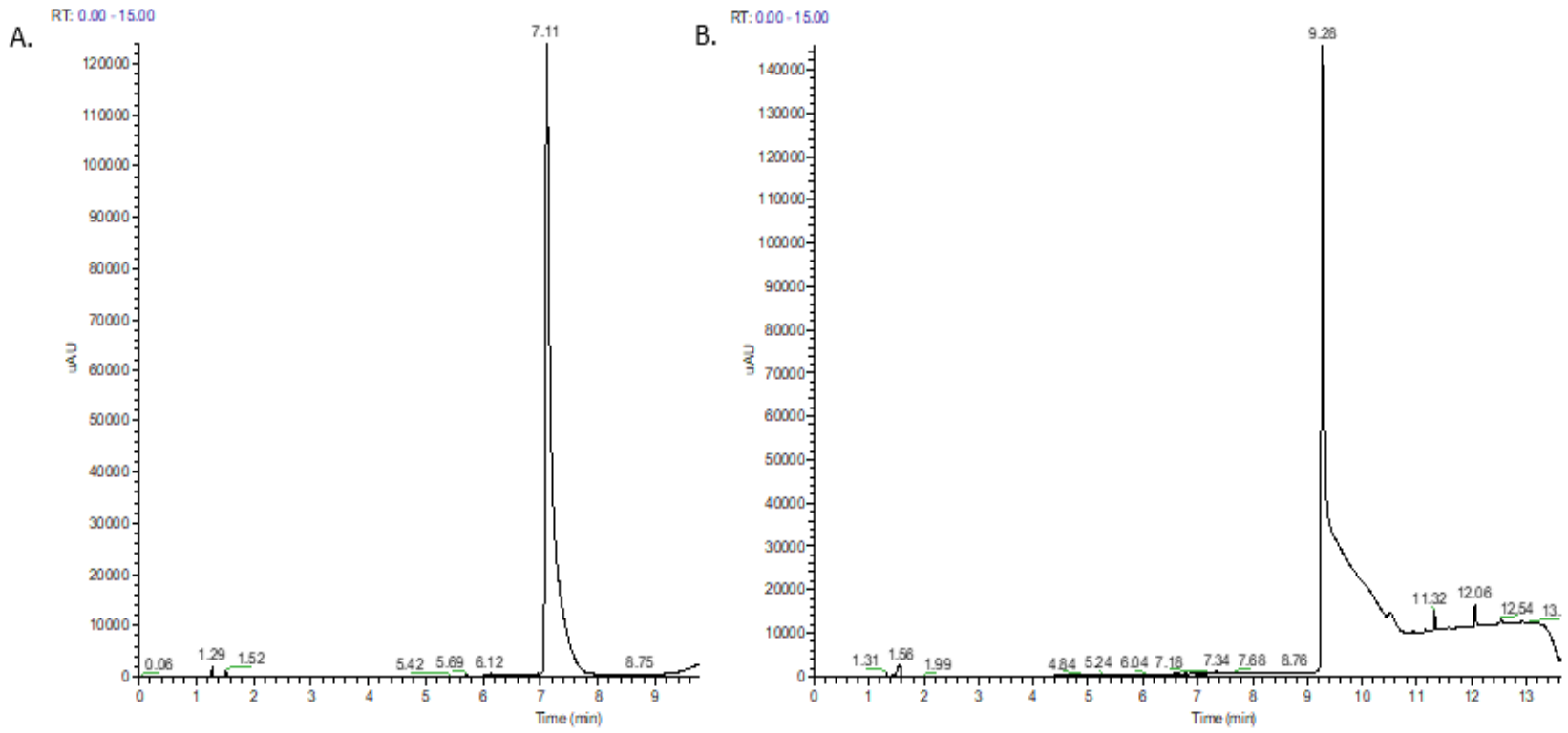

Figure 7. Representative UHPLC chromatogram of $100 \mu \mathrm{M}$ A) quercetin aglycone standard and B) kaempferol aglycone standard. 


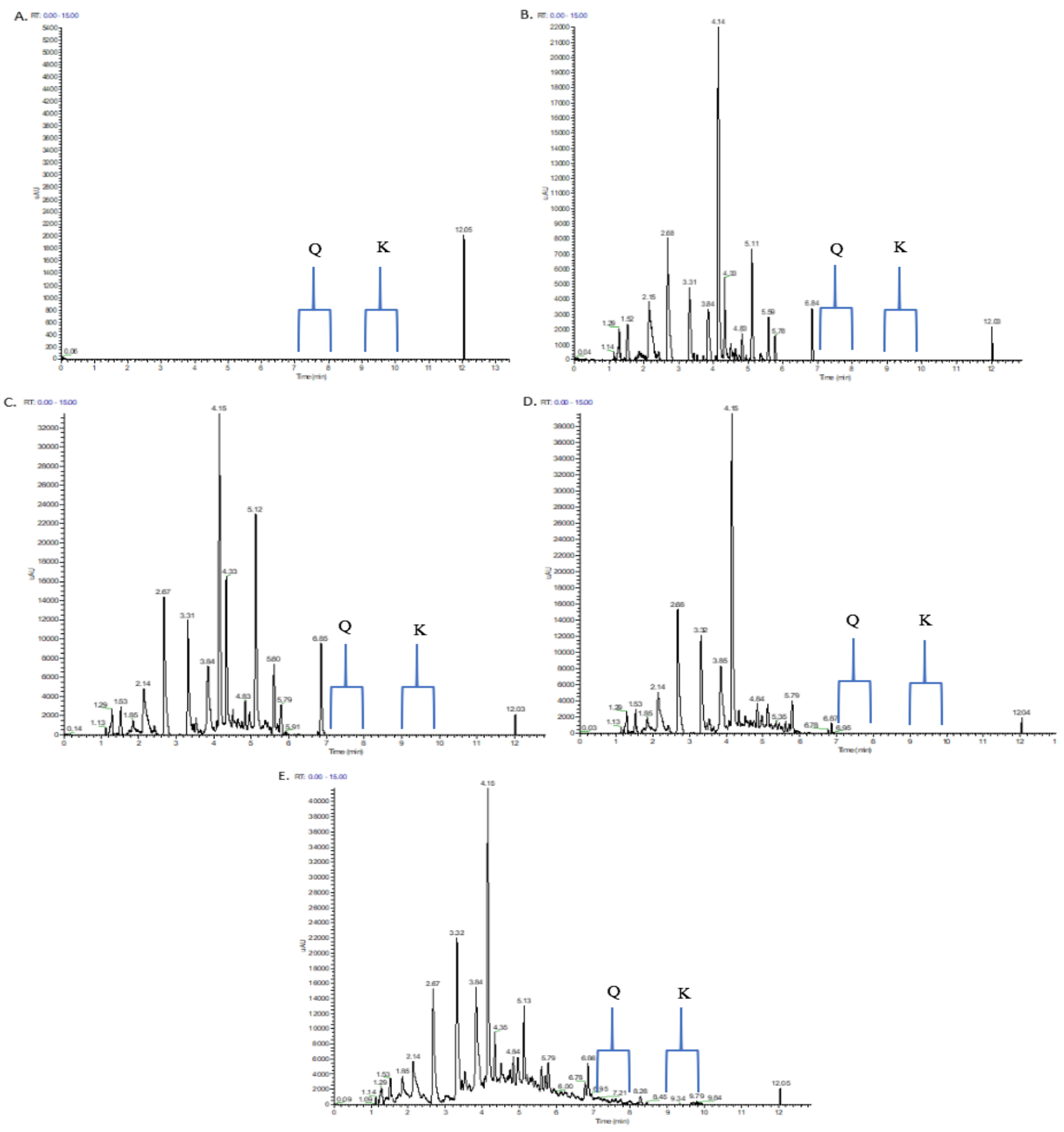

Figure 8. Representative UHPLC chromatogram of the hydrolysis of ramps tissues under different time intervals. A) hydrolysis for 2 hours, B) hydrolysis for 4 hours, C) hydrolysis for 6 hours, D) hydrolysis for 8 hours, E) hydrolysis for 12 hours. Q; detection region of quercetin aglycone, K; detection region of kaempferol aglycone. 
Hydrolysis of different types of starting material was also examined. Direct hydrolysis of plant tissues and glycosides extract shown in figure 9 resulted in the detection of quercetin and kaempferol aglycones after using the extract but not the plant tissues. Based on the $\mathrm{R}_{\mathrm{t}}$ of quercetin and kaempferol aglycone standards, hydrolysis of plant tissues resulted in no detection of hydrolyzed quercetin or kaempferol. However, hydrolysis of the extract showed a peak at $\mathrm{R}_{\mathrm{t}}$ 7.27 min indicating the hydrolysis of quercetin aglycone. Also, a second peak detected at $\mathrm{R}_{\mathrm{t}} 9.24$ min was indicated a peak corresponding to hydrolyzed kaempferol. The $\mathrm{R}_{\mathrm{t}}$ of both hydrolyzed quercetin and kaempferol from the hydrolysis of flavonol glycosides extract were slightly shifted downstream on the chromatogram. Although hydrolyzed quercetin and kaempferol were detected in the sample, the chromatogram indicated incomplete hydrolysis by $2 \mathrm{M} \mathrm{HCl}$ at $90^{\circ} \mathrm{C}$ for 2 hours. Thus, analysis of the effect of different $\mathrm{HCl}$ concentrations was conducted using flavonol glycosides extract.

A.

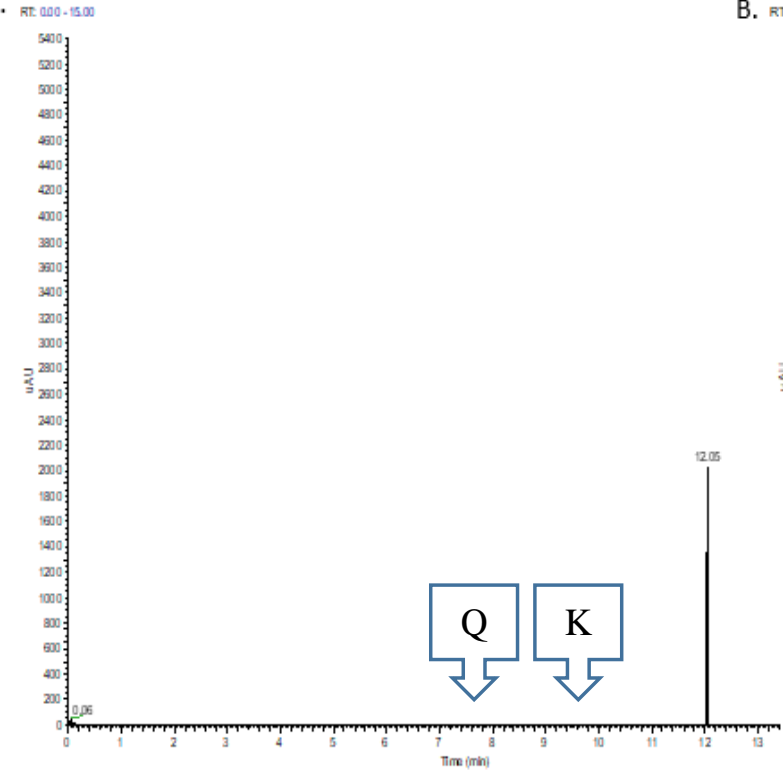

B. RT: $000 \cdot 15.00$

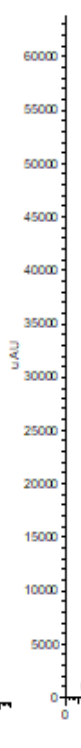

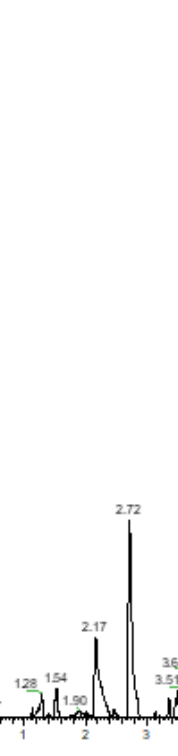

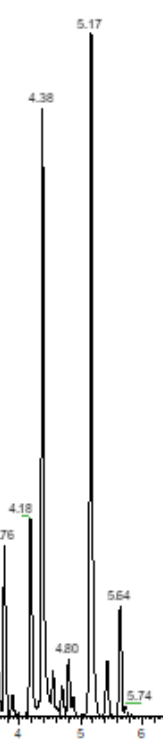

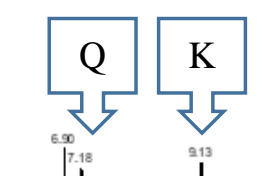
. 
Figure 10 shows a curve of hydrolyzed quercetin and kaempferol aglycone from flavonol glycosides extract under 1, 2, 3, 4, 5, and $6 \mathrm{M} \mathrm{HCl}$ at $90^{\circ} \mathrm{C}$ for 2 hours. The curve was calculated based on the area under the peak with Rt corresponding to each quercetin and kaempferol aglycone. The quercetin curve indicated that the optimal acid concentration that produced the highest intensity of hydrolyzed quercetin is at $3 \mathrm{M} \mathrm{HCl}$ with $\mathrm{R}_{\mathrm{t}}$ 7.19. Quercetin hydrolysis gradually declined with higher acid concentration. Kaempferol curve shows that the optimal acid concentration that produced the most intense peak at Rt 9.16 was $4 \mathrm{M} \mathrm{HCl}$. After 4M HCl, hydrolyzed kaempferol rapidly decreased with higher acid concentrations.

A.

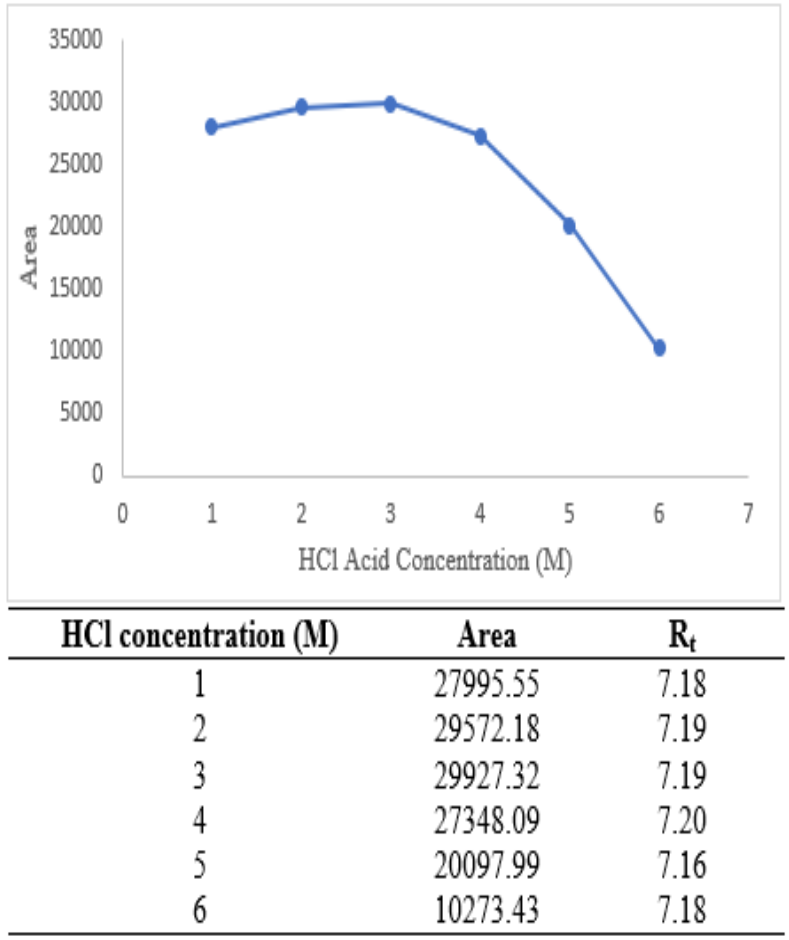

B.

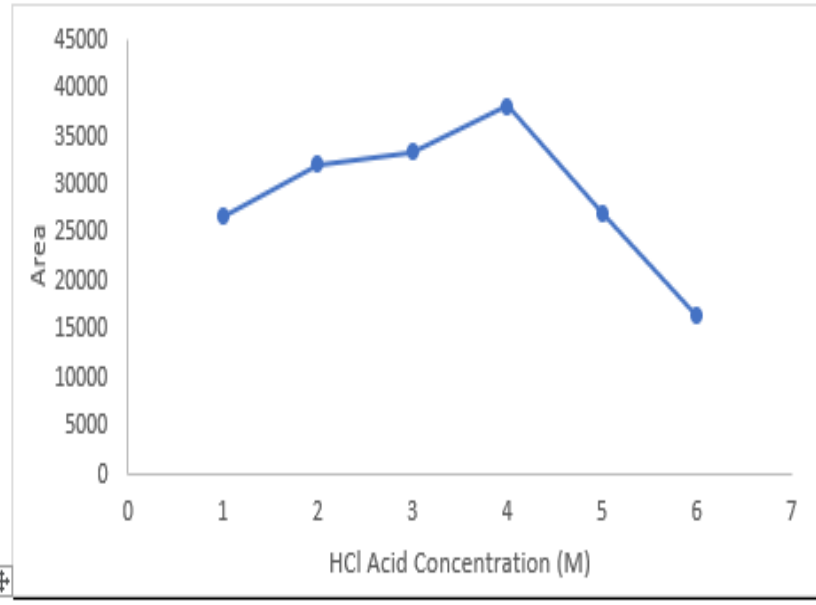

\begin{tabular}{ccc}
\hline HCl concentration $(M)$ & Area & $\mathbf{R}_{\mathbf{t}}$ \\
\hline 1 & 26712.27 & 9.13 \\
2 & 32145.61 & 9.15 \\
3 & 33413.7 & 9.14 \\
4 & 38128.53 & 9.16 \\
5 & 27159.38 & 9.13 \\
6 & 16402.52 & 9.14 \\
\hline
\end{tabular}

Figure 10. Results of the acid hydrolysis of flavonol glycosides under different $\mathrm{HCl}$ concentrations showing A) hydrolyzed quercetin aglycone, and B) hydrolyzed kaempferol aglycone. 


\section{CHAPTER 5: DISCUSSION}

To our knowledge, this is the first study to characterize major flavonol glycosides in whole ramps (Allium tricoccum) and individual parts. In this study, we found that the two major flavonol glycosides present in ramps were quercetin and kaempferol conjugates. Kaempferol glycoside was detected at a higher intensity than quercetin conjugate in whole ramps. All major quercetin and kaempferol conjugates were concentrated in leaves.

\section{A. Separation and Characterization of Major Flavanol Glycosides} Separation of Flavonol Glycosides

UHPLC protocol used to detect flavonol glycosides is specific to the analysis plant, properties of the desired compounds, and the chromatogram column. Therefore, UHPLC protocol was developed for the detection of flavonol glycosides in ramps. After examining the effect of changing the flow rate, a rate of $300 \mu \mathrm{L} /$ minute improved peaks separation. This finding is in agreement with other studies that detected flavonol glycosides using UHPLC under the same flow rate [99,107]. Increasing flow rate to $500 \mu \mathrm{L} /$ minute caused faster elution and shorter separation distance between the major peaks. The chromatogram of flavonol glycosides indicated that the two major peaks are highly polar, based on their early elution, thus, increasing the flow rate did not allow sufficient separation.

On the basis of flow rate analysis, $300 \mu \mathrm{L} /$ minute was used to examine the effect of gradient elution time for complete separation. An increase in the gradient elution time (90\% solvent A) to reach a total of 21 minutes, improved the separation of the major peaks because it allowed more time for the detection of polar compounds. Complete separation of all peaks was achieved by increasing the initial amount of solvent A to $98 \%$ and the elution time to a total of 24 minutes. The percentage and elution time of the polar solvent A is highly dependent on the 
polarity of the desired compounds [88]. In the unknown ramps extract, the detected flavonol glycosides are highly polar and thus optimal elution was achieved at higher concentration and elution time of the polar solvent A. This finding is consistent with the method used to detect flavonol glycosides in wild garlic [109]. Oszmianśki et al. analyzed flavonol glycosides with elution starting at $99 \%$ of the polar solvent A (aqueous solution $4.5 \%$ formic acid) which allowed the detection of kaempferol glycosides.

\section{Characterization of Major Glycosides In Ramps}

The separation method developed for the detection of flavonol glycosides was used to characterize the major glycosides in ramps. In this study, we found that the major flavonols in ramps were quercetin and kaempferol conjugates which is consistent with the flavonol content reported in the USDA flavonoid database in similar allium vegetables, spring onion and leeks [18]. The forms of quercetin and kaempferol conjugates detected by UHPLC-PDA-MS ${ }^{2}$ in ramps were putatively kaempferol sophoroside glucuronide, quercetin sophoroside glucuronide, and quercetin dihexoside. Although analysis of the closely related wild garlic showed the presence of kaempferol conjugates, the sugar moieties linked to kaempferol backbone were different than in ramps. Kaempferol in wild garlic was found to be linked to the disaccharides glucose pyranoside and neohesperidoside [3]. However, the characterization of major quercetin and kaempferol glycosides in ramps was consistant with conjugates detected in onion [106].

Although quercetin dihexoside was identified in ramps, the particular type and connection of the hexosides in can't be determined using UHPLC-PDA-MS² detection method. Major quercetin and kaempferol conjugates contained sophoroside as the dihexoside. The existance of sophorose in quercetin sophoroside glucuronide may indicate that the second 
quercetin dihexoside conjugate is a sophoroside which might be an intermediate in the synthesis reaction.

Analysis of the major flavonol glycosides in individual parts indicated that flavonol glycosides were concentrated in leaves. The concentration of kaempferol sophoroside glucuronide was higher than the quercetin conjugates in leaves. This result is consistent with Nuutila et al. findings that showed higher kaempferol concentration in the green leafy part of spring onion (Allium cepa) and spinach (Spinacia oleracea) [23]. However, the absence of flavonol glycosides, specifically quercetin, from the bulb was unexpected because several reports showed that quercetin was high in onion bulb $[17,23,93]$. Conversely, quercetin content in leeks, closely similar to ramps, reported in the USDA flavonoids database was low $(0.09 \mathrm{mg} / 100 \mathrm{~g})$. ${ }^{1} \mathrm{H}$ and ${ }^{13} \mathrm{C}$ NMR Preliminary Analysis

Analysis of flavonol glycoside extract by NMR confirmed the existence of the glycosides conjugates and flavonol backbones. Although the spectra showed a typical spectral regions for the different skeletal units of proposed structures: kaemperol sophoroside glucuronide (MW, 786), quercetin sophoroside glucuronide (MW, 802), and quercetin dihexoside (MW, 626), the specific attachment positions and complete structural elucidation was not achieved. This limitation was due to the use of a crude flavonol glycoside mixture containing different interfering compounds extracted from plant tissues. In order to conduct a complete structural elucidation of the major flavonol glycosides in ramps, an isolation of individual compounds is required. 


\section{B. Preliminary Development of Acid Hydrolysis Protocol for Quantification of Flavonol}

\section{Aglycones}

Results from the preliminary quantification protocol showed that maximum hydrolysis was achieved by hydrolyzing flavonol glycosides extract in $3 \mathrm{M} \mathrm{HCl}$ at $90^{\circ} \mathrm{C}$ for two hours. No detection of the aglycones was observed when pulverized ramps was hydrolyzed by $1.2 \mathrm{M} \mathrm{HCl}$ under different time intervals at $80^{\circ} \mathrm{C}$. This might be due to the type of starting material used. In this experiment, direct hydrolysis of ramps tissues was used. Ramps tissues contain many different components with varying polarities that may have neutralized the acid and interfered with the hydrolysis of glycosides. Thus, hydrolysis of different starting material was also examined.

Quercetin and kaempferol aglycones were detected after the hydrolysis of flavonol glycosides extract. This finding was consistent with other studies that used glycosides extract as a starting material $[104,110]$. The elimination of other interfering plant tissues and components by extraction of flavonol glycosides allowed a direct exposure of the glycosides to the acid. Nuutila et al, however, optimized method for flavonol quantification using direct hydrolysis of flavonol glycosides in onions [23]. This might be explained by the differences in composition of onions and ramps. Flavonol glycosides in ramps are more complex and polar than onions thus, need stronger hydrolysis conditions.

On the basis of results from different starting material, the effect of different acid concentrations was examined. The hydrolysis of glycosides' extract under acid concentrations (1-6M HCl) showed that highest intensity of quercetin aglycone was achieved at 3M $\mathrm{HCl}$. Maximum hydrolysis of kaempferol, however, was obtained under 4M HCl. Optimization of flavonol glycosides hydrolysis from broccoli extract indicated that $2 \mathrm{M} \mathrm{HCl}$ resulted in the 
detection of the highest amount of quercetin and kaempferol aglycone [105]. The decline in quercetin and kaempferol aglycones observed under higher acid concentration can be related to the degradation of hydrolyzed aglycone by the acid. Hertog et al reported a degradation of hydrolyzed flavonols under higher acid conditions [111]. Also, optimum conditions for the consistent hydrolysis of flavonol glycosides in leeks, onion, and cranberry was challenging because it is dependent on the type of different sugar moieties connected. The presence of extra hydroxyl group on quercetin aglycone relative to kaempferol increases the reactivity of quercetin with the added acid; thus, optimum quercetin hydrolysis was obtained under lower acid concentration compared to kaempferol. 


\section{CHAPTER 6: CONCLUSION}

To our knowledge, this work marks the first qualitative analysis conducted for the detection of major flavonol glycosides content in ramps. The main findings revealed that quercetin and kaempferol conjugates are the major flavonols in ramps and that the flavonols are located specifically in the leaves of the plant. The identification of flavonol conjugates provides a potential rationale for the reported health benefits of ramps in Appalachian folk medicine. Future studies are needed to completely isolate the identified flavonol glycosides for a detailed structural elucidation. Also, it is essential to quantify total flavonols in ramps to determine the effective dosage needed to be consumed in the diet for optimal health benefits. 


\section{REFERENCES}

1. How to Grow Ramps, the Wild Leek Available online:

https://www.thespruce.com/growing-ramps-in-the-vegetable-garden-1403463 (accessed on Mar 12, 2019).

2. Sengupta, A.; Ghosh, S.; Bhattacharjee, S. Allium vegetables in cancer prevention: an overview. Asian Pac. J. Cancer Prev. 2004, 5, 237-245.

3. Carotenuto, A.; De Feo, V.; Fattorusso, E.; Lanzotti, V.; Magno, S.; Cicala, C. The flavonoids of Allium ursinum. Phytochemistry 1996, 41, 531-536.

4. Chamberlain, J.; Beegle, D.; Connette, K. Forest Farming Ramps- Agroforestry Notes. National Agroforestry Center 2014, 8.

5. Cultivation of Ramps (Allium tricoccum and A. burdickii) | NC State Extension Publications Available online: https://content.ces.ncsu.edu/cultivation-of-ramps-alliumtricoccum-and-a-burdickii (accessed on Nov 4, 2018).

6. A Field Guide to Medicinal Plants: Eastern and Central North America (Book). Science News 1990, 137, 402-402.

7. Cavender, A. Folk medical uses of plant foods in southern Appalachia, United States. Journal of Ethnopharmacology 2006, 108, 74-84.

8. Calvey, E.M.; White, K.D.; Matusik, J.E.; Sha, D.; Block, E. Allium chemistry: identification of organosulfur compounds in ramp (Allium tricoccum) homogenates. Phytochemistry 1998, 49, 359-364.

9. Zennie, T.; Ogzewalla, D. Ascorbic acid and Vitamin A content of edible wild plants of Ohio and Kentucky. Economic Botany 1977, 31, 76-79.

10. Preuss, H.G.; Clouatre, D.; Mohamadi, A.; Jarrell, S.T. Wild garlic has a greater effect than regular garlic on blood pressure and blood chemistries of rats. Int Urol Nephrol 2001, 32, 525-530.

11. Badary, O.A.; Yassin, N.A.Z.; El-Shenawy, S.M.A.; EL-Moneem, M.A.; AL-Shafeiy, H.M. STUDY OF THE EFFECT OF Allium porrum. 2013, 12.

12. Zeng, Y.; Li, Y.; Yang, J.; Pu, X.; Du, J.; Yang, X.; Yang, T.; Yang, S. Therapeutic Role of Functional Components in Alliums for Preventive Chronic Disease in Human Being. Evid Based Complement Alternat Med 2017, 2017.

13. Falcone Ferreyra, M.L.; Rius, S.P.; Casati, P. Flavonoids: biosynthesis, biological functions, and biotechnological applications. Front Plant Sci 2012, 3.

14. Kumar, S.; Pandey, A.K. Chemistry and Biological Activities of Flavonoids: An Overview. ScientificWorldJournal 2013, 2013.

15. Manach, C.; Scalbert, A.; Morand, C.; Rémésy, C.; Jiménez, L. Polyphenols: food sources and bioavailability. Am J Clin Nutr 2004, 79, 727-747.

16. Fattorusso, E.; Lanzotti, V.; Taglialatela-Scafati, O.; Cicala, C. The flavonoids of leek, Allium porrum. Phytochemistry 2001, 57, 565-569.

17. Crozier, A.; Lean, M.E.J.; McDonald, M.S.; Black, C. Quantitative Analysis of the Flavonoid Content of Commercial Tomatoes, Onions, Lettuce, and Celery. Journal of Agricultural and Food Chemistry 1997, 45, 590-595.

18. Bhagwat, S.; Haytowitz, D.B.; Holden, J.M. USDA Database for the Flavonoid Content of Selected Foods Release 3. 176.

19. Vuorinen, H.; Määtta, K.; Törrönen, R. Content of the flavonols myricetin, quercetin, and kaempferol in finnish berry wines. J. Agric. Food Chem. 2000, 48, 2675-2680. 
20. McDonald, null; Hughes, null; Burns, null; Lean, null; Matthews, null; Crozier, null Survey of the Free and Conjugated Myricetin and Quercetin Content of Red Wines of Different Geographical Origins. J. Agric. Food Chem. 1998, 46, 368-375.

21. Lakenbrink, C.; Lapczynski, S.; Maiwald, B.; Engelhardt, U.H. Flavonoids and other polyphenols in consumer brews of tea and other caffeinated beverages. J. Agric. Food Chem. 2000, 48, 2848-2852.

22. Aherne, S.A.; O’Brien, N.M. Dietary flavonols: chemistry, food content, and metabolism. Nutrition 2002, 18, 75-81.

23. Nuutila, A.M.; Kammiovirta, K.; Oksman-Caldentey, K.-M. Comparison of methods for the hydrolysis of flavonoids and phenolic acids from onion and spinach for HPLC analysis. Food Chemistry 2002, 76, 519-525.

24. Smith, C.; Lombard, K.A.; Peffley, E.B.; Liu, W. Genetic Analysis of Quercetin in Onion (Allium cepa L.) “Lady Raider.” Texas Journal of Agriculture and Natural Resources 2016, 16, 24-28.

25. Morris, M.C.; Wang, Y.; Barnes, L.L.; Bennett, D.A.; Dawson-Hughes, B.; Booth, S.L. Nutrients and bioactives in green leafy vegetables and cognitive decline: Prospective study. Neurology 2018, 90, e214-e222.

26. Hollman, P.C.H. Absorption, Bioavailability, and Metabolism of Flavonoids. Pharmaceutical Biology 2004, 42, 74-83.

27. Xiao, J. Dietary flavonoid aglycones and their glycosides: Which show better biological significance? Crit Rev Food Sci Nutr 2017, 57, 1874-1905.

28. Lu, X.; Ross, C.F.; Powers, J.R.; Rasco, B.A. Determination of Quercetins in Onion (Allium cepa) Using Infrared Spectroscopy. J. Agric. Food Chem. 2011, 59, 6376-6382.

29. Ramps In West Virginia Available online: https://wildwestvirginiaramps.com/ramps-westvirginia/ (accessed on Jul 9, 2019).

30. Radovanović, B.; Mladenović, J.; Radovanović, A.; Pavlović, R.; Nikolić, V. Phenolic Composition, Antioxidant, Antimicrobial and Cytotoxic Activites of Allium porrum L. (Serbia) Extracts. Journal of Food and Nutrition Research 2015, 6.

31. Pejatović, T.; Samardžić, D.; Krivokapić, S. Antioxidative properities of a traditional tincture and several leaf extracts of Allium ursinum L. (collected in Montenegro and Bosnia and Herzegovina). 2017, 6.

32. Bourgaud, F.; Gravot, A.; Milesi, S.; Gontier, E. Production of plant secondary metabolites: a historical perspective. Plant Science 2001, 161, 839-851.

33. Panche, A.N.; Diwan, A.D.; Chandra, S.R. Flavonoids: an overview. J Nutr Sci 2016, 5.

34. Plumb, J.; Pigat, S.; Bompola, F.; Cushen, M.; Pinchen, H.; Nørby, E.; Astley, S.; Lyons, J.; Kiely, M.; Finglas, P. eBASIS (Bioactive Substances in Food Information Systems) and Bioactive Intakes: Major Updates of the Bioactive Compound Composition and Beneficial Bioeffects Database and the Development of a Probabilistic Model to Assess Intakes in Europe. Nutrients 2017, 9.

35. Neveu, V.; Perez-Jiménez, J.; Vos, F.; Crespy, V.; du Chaffaut, L.; Mennen, L.; Knox, C.; Eisner, R.; Cruz, J.; Wishart, D.; et al. Phenol-Explorer: an online comprehensive database on polyphenol contents in foods. Database (Oxford) 2010, 2010.

36. Read "Dietary Reference Intakes: Proposed Definition and Plan for Review of Dietary Antioxidants and Related Compounds" at NAP.edu;

37. Chun, O.K.; Chung, S.J.; Song, W.O. Estimated Dietary Flavonoid Intake and Major Food Sources of U.S. Adults. J Nutr 2007, 137, 1244-1252. 
38. Cassidy, A.; O’Reilly, É.J.; Kay, C.; Sampson, L.; Franz, M.; Forman, J.; Curhan, G.; Rimm, E.B. Habitual intake of flavonoid subclasses and incident hypertension in adults123. Am J Clin Nutr 2011, 93, 338-347.

39. Kesse-Guyot, E.; Fezeu, L.; Andreeva, V.A.; Touvier, M.; Scalbert, A.; Hercberg, S.; Galan, P. Total and Specific Polyphenol Intakes in Midlife Are Associated with Cognitive Function Measured 13 Years Later. J Nutr 2012, 142, 76-83.

40. Zamora-Ros, R.; Knaze, V.; Luján-Barroso, L.; Slimani, N.; Romieu, I.; Fedirko, V.; Magistris, M.S. de; Ericson, U.; Amiano, P.; Trichopoulou, A.; et al. Estimated dietary intakes of flavonols, flavanones and flavones in the European Prospective Investigation into Cancer and Nutrition (EPIC) 24 hour dietary recall cohort. British Journal of Nutrition 2011, 106, 1915-1925.

41. Bai, W.; Wang, C.; Ren, C. Intakes of total and individual flavonoids by US adults. Int $J$ Food Sci Nutr 2014, 65, 9-20.

42. Scalbert, A.; Williamson, G. Dietary intake and bioavailability of polyphenols. J. Nutr. 2000, 130, 2073S-85S.

43. Cao, J.; Chen, W.; Zhang, Y.; Zhang, Y.; Zhao, X. Content of Selected Flavonoids in 100 Edible Vegetables and Fruits. Food Science and Technology Research 2010, 16, 395-402.

44. Sharma, A.; Sharma, P.; Tuli, H.S.; Sharma, A.K. Phytochemical and Pharmacological Properties of Flavonols. In eLS; American Cancer Society, 2018; pp. 1-12 ISBN 978-0470-01590-2.

45. Jiang, H.; Engelhardt, U.H.; Thräne, C.; Maiwald, B.; Stark, J. Determination of flavonol glycosides in green tea, oolong tea and black tea by UHPLC compared to HPLC. Food Chem 2015, 183, 30-35.

46. Xiao, J.; Muzashvili, T.S.; Georgiev, M.I. Advances in the biotechnological glycosylation of valuable flavonoids. Biotechnol. Adv. 2014, 32, 1145-1156.

47. Thilakarathna, S.H.; Rupasinghe, H.P.V. Flavonoid Bioavailability and Attempts for Bioavailability Enhancement. Nutrients 2013, 5, 3367-3387.

48. Chow, S.-C. Bioavailability and Bioequivalence in Drug Development. Wiley Interdiscip Rev Comput Stat 2014, 6, 304-312.

49. Rein, M.J.; Renouf, M.; Cruz-Hernandez, C.; Actis-Goretta, L.; Thakkar, S.K.; da Silva Pinto, M. Bioavailability of bioactive food compounds: a challenging journey to bioefficacy. Br J Clin Pharmacol 2013, 75, 588-602.

50. Williamson, G.; Kay, C.D.; Crozier, A. The Bioavailability, Transport, and Bioactivity of Dietary Flavonoids: A Review from a Historical Perspective. Comprehensive Reviews in Food Science and Food Safety 2018, 17, 1054-1112.

51. Alvarez, A.I.; Real, R.; Pérez, M.; Mendoza, G.; Prieto, J.G.; Merino, G. Modulation of the activity of ABC transporters (P-glycoprotein, MRP2, BCRP) by flavonoids and drug response. Journal of Pharmaceutical Sciences 2010, 99, 598-617.

52. Day, A.J.; DuPont, M.S.; Ridley, S.; Rhodes, M.; Rhodes, M.J.C.; Morgan, M.R.A.; Williamson, G. Deglycosylation of flavonoid and isoflavonoid glycosides by human small intestine and liver $\beta$-glucosidase activity. FEBS Letters 1998, 436, 71-75.

53. Graefe, E.U.; Wittig, J.; Mueller, S.; Riethling, A.-K.; Uehleke, B.; Drewelow, B.; Pforte, H.; Jacobasch, G.; Derendorf, H.; Veit, M. Pharmacokinetics and Bioavailability of Quercetin Glycosides in Humans. The Journal of Clinical Pharmacology 2001, 41, 492499. 
54. Németh, K.; Plumb, G.W.; Berrin, J.-G.; Juge, N.; Jacob, R.; Naim, H.Y.; Williamson, G.; Swallow, D.M.; Kroon, P.A. Deglycosylation by small intestinal epithelial cell betaglucosidases is a critical step in the absorption and metabolism of dietary flavonoid glycosides in humans. Eur J Nutr 2003, 42, 29-42.

55. Walgren, R.A.; Lin, J.T.; Kinne, R.K.; Walle, T. Cellular uptake of dietary flavonoid quercetin 4'-beta-glucoside by sodium-dependent glucose transporter SGLT1. $J$.

Pharmacol. Exp. Ther. 2000, 294, 837-843.

56. Manach, C.; Regerat, F.; Texier, O.; Agullo, G.; Demigne, C.; Remesy, C. Bioavailability, metabolism and physiological impact of 4-oxo-flavonoids. Nutrition Research 1996, 16, 517-544.

57. O’Leary, K.A.; Day, A.J.; Needs, P.W.; Mellon, F.A.; O’Brien, N.M.; Williamson, G. Metabolism of quercetin-7- and quercetin-3-glucuronides by an in vitro hepatic model: the role of human beta-glucuronidase, sulfotransferase, catechol-O-methyltransferase and multi-resistant protein 2 (MRP2) in flavonoid metabolism. Biochem. Pharmacol. 2003, 65, 479-491.

58. Marín, L.; Miguélez, E.M.; Villar, C.J.; Lombó, F. Bioavailability of Dietary Polyphenols and Gut Microbiota Metabolism: Antimicrobial Properties Available online: https://www.hindawi.com/journals/bmri/2015/905215/ (accessed on Jan 31, 2019).

59. Lu, Q.-Y.; Zhang, L.; Eibl, G.; Go, V.L.W. Overestimation of flavonoid aglycones as a result of the ex vivo deconjugation of glucuronides by the tissue $\beta$-glucuronidase. $J$ Pharm Biomed Anal 2014, 88, 364-369.

60. Mullen, W.; Edwards, C.A.; Crozier, A. Absorption, excretion and metabolite profiling of methyl-, glucuronyl-, glucosyl- and sulpho-conjugates of quercetin in human plasma and urine after ingestion of onions. British Journal of Nutrition 2006, 96, 107.

61. Serra, A.; Macià, A.; Romero, M.-P.; Reguant, J.; Ortega, N.; Motilva, M.-J. Metabolic pathways of the colonic metabolism of flavonoids (flavonols, flavones and flavanones) and phenolic acids. Food Chemistry 2012, 130, 383-393.

62. Mullen, W.; Rouanet, J.-M.; Auger, C.; Teissèdre, P.-L.; Caldwell, S.T.; Hartley, R.C.; Lean, M.E.J.; Edwards, C.A.; Crozier, A. Bioavailability of [2-(14)C]quercetin-4'glucoside in rats. J. Agric. Food Chem. 2008, 56, 12127-12137.

63. Viskupicova, J.; Ondrejovič, M.; Sturdik, E. Bioavailability and metabolism of flavonoids. Journal of food and nutrition research 2008, 47, 151-162.

64. Vacek, J.; Papoušková, B.; Kosina, P.; Vrba, J.; Křen, V.; Ulrichová, J. Biotransformation of flavonols and taxifolin in hepatocyte in vitro systems as determined by liquid chromatography with various stationary phases and electrospray ionization-quadrupole time-of-flight mass spectrometry. Journal of Chromatography B 2012, 899, 109-115.

65. Lee, H.N.; Shin, S.A.; Choo, G.S.; Kim, H.J.; Park, Y.S.; Kim, B.S.; Kim, S.K.; Cho, S.D.; Nam, J.S.; Choi, C.S.; et al. Anti-inflammatory effect of quercetin and galangin in LPSstimulated RAW264.7 macrophages and DNCB-induced atopic dermatitis animal models. Int J Mol Med 2018, 41, 888-898.

66. Egert, S.; Bosy-Westphal, A.; Seiberl, J.; Kürbitz, C.; Settler, U.; Plachta-Danielzik, S.; Wagner, A.E.; Frank, J.; Schrezenmeir, J.; Rimbach, G.; et al. Quercetin reduces systolic blood pressure and plasma oxidised low-density lipoprotein concentrations in overweight subjects with a high-cardiovascular disease risk phenotype: a double-blinded, placebocontrolled cross-over study. The British Journal of Nutrition; Cambridge 2009, 102, 106574. 
67. Edwards, R.L.; Lyon, T.; Litwin, S.E.; Rabovsky, A.; Symons, J.D.; Jalili, T. Quercetin reduces blood pressure in hypertensive subjects. J. Nutr. 2007, 137, 2405-2411.

68. Crespo, I.; García-Mediavilla, M.V.; Gutiérrez, B.; Sánchez-Campos, S.; Tuñón, M.J.; González-Gallego, J. A comparison of the effects of kaempferol and quercetin on cytokineinduced pro-inflammatory status of cultured human endothelial cells. British Journal of Nutrition 2008, 100, 968-976.

69. Sanz, M.J.; Ferrandiz, M.L.; Cejudo, M.; Terencio, M.C.; Gil, B.; Bustos, G.; Ubeda, A.; Gunasegaran, R.; Alcaraz, M.J. Influence of a series of natural flavonoids on free radical generating systems and oxidative stress. Xenobiotica 1994, 24, 689-699.

70. Kampkötter, A.; Gombitang Nkwonkam, C.; Zurawski, R.F.; Timpel, C.; Chovolou, Y.; Wätjen, W.; Kahl, R. Effects of the flavonoids kaempferol and fisetin on thermotolerance, oxidative stress and FoxO transcription factor DAF-16 in the model organism Caenorhabditis elegans. Arch. Toxicol. 2007, 81, 849-858.

71. Wang, J.; Fang, X.; Ge, L.; Cao, F.; Zhao, L.; Wang, Z.; Xiao, W. Antitumor, antioxidant and anti-inflammatory activities of kaempferol and its corresponding glycosides and the enzymatic preparation of kaempferol. PLOS ONE 2018, 13.

72. Devi, K.P.; Malar, D.S.; Nabavi, S.F.; Sureda, A.; Xiao, J.; Nabavi, S.M.; Daglia, M. Kaempferol and inflammation: From chemistry to medicine. Pharmacological Research 2015, 99, 1-10.

73. Lin, J.; Rexrode, K.M.; Hu, F.; Albert, C.M.; Chae, C.U.; Rimm, E.B.; Stampfer, M.J.; Manson, J.E. Dietary Intakes of Flavonols and Flavones and Coronary Heart Disease in US Women. Am J Epidemiol 2007, 165, 1305-1313.

74. Geleijnse, J.M.; Launer, L.J.; Van der Kuip, D.A.M.; Hofman, A.; Witteman, J.C.M. Inverse association of tea and flavonoid intakes with incident myocardial infarction: the Rotterdam Study. Am. J. Clin. Nutr. 2002, 75, 880-886.

75. Marniemi, J.; Alanen, E.; Impivaara, O.; Seppänen, R.; Hakala, P.; Rajala, T.; Rönnemaa, T. Dietary and serum vitamins and minerals as predictors of myocardial infarction and stroke in elderly subjects. Nutr Metab Cardiovasc Dis 2005, 15, 188-197.

76. Iwashina, T. The Structure and Distribution of the Flavonoids in Plants. J Plant Res 2000, 113, 287-299.

77. Tanaka, Y.; Sasaki, N.; Ohmiya, A. Biosynthesis of plant pigments: anthocyanins, betalains and carotenoids. Plant J. 2008, 54, 733-749.

78. Treutter, D. Significance of flavonoids in plant resistance and enhancement of their biosynthesis. Plant Biol (Stuttg) 2005, 7, 581-591.

79. Martinez, V.; Mestre, T.C.; Rubio, F.; Girones-Vilaplana, A.; Moreno, D.A.; Mittler, R.; Rivero, R.M. Accumulation of Flavonols over Hydroxycinnamic Acids Favors Oxidative Damage Protection under Abiotic Stress. Front. Plant Sci. 2016, 7.

80. Stefanowska, M.; Kuraś, M.; Kacperska, A. Low temperature-induced modifications in cell ultrastructure and localization of phenolics in winter oilseed rape (Brassica napus L. var. oleifera L.) leaves. Ann. Bot. 2002, 90, 637-645.

81. Smeets, K.; Van Damme, E.J.M.; Van Leuven, F.; Peumans, W.J. Isolation, characterization and molecular cloning of a leaf-specific lectin from ramsons (Allium ursinum L.). Plant Mol Biol 1997, 35, 531-535.

82. Awouafack, M.D.; Tane, P.; Morita, H. Isolation and Structure Characterization of Flavonoids. Flavonoids - From Biosynthesis to Human Health 2017. 
83. Do, Q.D.; Angkawijaya, A.E.; Tran-Nguyen, P.L.; Huynh, L.H.; Soetaredjo, F.E.; Ismadji, S.; Ju, Y.-H. Effect of extraction solvent on total phenol content, total flavonoid content, and antioxidant activity of Limnophila aromatica. Journal of Food and Drug Analysis 2014, 22, 296-302.

84. Kim, D.-O.; Chun, O.K.; Kim, Y.J.; Moon, H.-Y.; Lee, C.Y. Quantification of Polyphenolics and Their Antioxidant Capacity in Fresh Plums. J. Agric. Food Chem. 2003, 51, 6509-6515.

85. Kovinich, N.; Saleem, A.; Arnason, J.T.; Miki, B. Combined analysis of transcriptome and metabolite data reveals extensive differences between black and brown nearly-isogenic soybean (Glycine max) seed coats enabling the identification of pigment isogenes. BMC Genomics 2011, 12.

86. A Review on the Extraction Methods Use in Medicinal Plants, Principle, Strength and Limitation. Medicinal \& Aromatic Plants 2015, 04.

87. Rabeta, M.S.; Lin, S.P. Effects of Different Drying Methods on the Antioxidant Activities of Leaves and Berries of Cayratia trifolia. Sains Malaysiana 2015, 44, 275-280.

88. Liquid Chromatography Available online:

https://chem.libretexts.org/Bookshelves/Analytical_Chemistry/Supplemental_Modules_(An alytical_Chemistry)/Instrumental_Analysis/Chromatography/Liquid_Chromatography (accessed on Jul 7, 2019).

89. Bajpai, V.K.; Majumder, R.; Park, J.G. Isolation and purification of plant secondary metabolites using column-chromatographic technique.; 2016.

90. Coskun, O. Separation techniques: Chromatography. North Clin Istanb 2016, 3, 156-160.

91. Pellegrini, N.; Colombi, B.; Salvatore, S.; Brenna, O.V.; Galaverna, G.; Rio, D.D.; Bianchi, M.; Bennett, R.N.; Brighenti, F. Evaluation of antioxidant capacity of some fruit and vegetable foods: efficiency of extraction of a sequence of solvents. Journal of the Science of Food and Agriculture 2007, 87, 103-111.

92. Hohenstein, J.D.; Studham, M.E.; Klein, A.; Kovinich, N.; Barry, K.; Lee, Y.-J.; MacIntosh, G.C. Transcriptional and Chemical Changes in Soybean Leaves in Response to Long-Term Aphid Colonization. Front Plant Sci 2019, 10.

93. Caridi, D.; Trenerry, V.C.; Rochfort, S.; Duong, S.; Laugher, D.; Jones, R. Profiling and quantifying quercetin glucosides in onion (Allium cepa L.) varieties using capillary zone electrophoresis and high performance liquid chromatography. Food Chemistry 2007, 105, 691-699.

94. Vvedenskaya, I.O.; Rosen, R.T.; Guido, J.E.; Russell, D.J.; Mills, K.A.; Vorsa, N. Characterization of Flavonols in Cranberry (Vaccinium macrocarpon) Powder. J. Agric. Food Chem. 2004, 52, 188-195.

95. Vrhovsek, U.; Masuero, D.; Palmieri, L.; Mattivi, F. Identification and quantification of flavonol glycosides in cultivated blueberry cultivars. Journal of Food Composition and Analysis 2012, 25, 9-16.

96. Cuyckens, F.; Claeys, M. Mass spectrometry in the structural analysis of flavonoids. Journal of Mass Spectrometry 2004, 39, 1-15.

97. Fulcrand, H.; Mané, C.; Preys, S.; Mazerolles, G.; Bouchut, C.; Mazauric, J.-P.; Souquet, J.-M.; Meudec, E.; Li, Y.; Cole, R.B.; et al. Direct mass spectrometry approaches to characterize polyphenol composition of complex samples. Phytochemistry 2008, 69, 31313138. 
98. Vijlder, T.D.; Valkenborg, D.; Lemière, F.; Romijn, E.P.; Laukens, K.; Cuyckens, F. A tutorial in small molecule identification via electrospray ionization-mass spectrometry: The practical art of structural elucidation. Mass Spectrometry Reviews 2018, 37, 607-629.

99. Jang, G.H.; Kim, H.W.; Lee, M.K.; Jeong, S.Y.; Bak, A.R.; Lee, D.J.; Kim, J.B.

Characterization and quantification of flavonoid glycosides in the Prunus genus by UPLC-

DAD-QTOF/MS. Saudi Journal of Biological Sciences 2018, 25, 1622-1631.

100. NMR Spectroscopy Available online:

https://www2.chemistry.msu.edu/faculty/reusch/VirtTxtJml/Spectrpy/nmr/nmr1.htm (accessed on Jul 8, 2019).

101. Savage, A.K.; van Duynhoven, J.P.M.; Tucker, G.; Daykin, C.A. Enhanced NMR-based profiling of polyphenols in commercially available grape juices using solid-phase extraction. Magn Reson Chem 2011, 49 Suppl 1, S27-36.

102. Shahat, A.A.; Abdelshafeek, K.A.; Husseiny, H.A. Isolation and identification of a new flavonoid glycoside from Carrichtera annua L. seeds. Pharmacognosy Res 2011, 3, 151154.

103. Harbaum, B.; Hubbermann, E.M.; Wolff, C.; Herges, R.; Zhu, Z.; Schwarz, K. Identification of flavonoids and hydroxycinnamic acids in pak choi varieties (Brassica campestris L. ssp. chinensis var. communis) by HPLC-ESI-MSn and NMR and their quantification by HPLC-DAD. J. Agric. Food Chem. 2007, 55, 8251-8260.

104. Plazonic, A.; Males, Z.; Mornar, A.; Nigović, B.; Kujundzic, N. Characterization and quantification of flavonoid aglycones and phenolic acids in the hydrolyzed methanolic extract of Caucalis platycarpos using HPLC-DAD-MS/MS. Chemistry of Natural Compounds 2010, 47, 27-32.

105. Tuszyńska, M. Validation of the Analytical Method for the Determination of Flavonoids in Broccoli.; 2014.

106. Urushibara, S.; Kitayama, Y.; Watanabe, T.; Okuno, T.; Watarai, A.; Matsumoto, T. New flavonol glycosides, major determinants inducing the green fluorescence in the guard cells of allium cepa. Tetrahedron Letters 1992, 33, 1213-1216.

107. Lin, L.-Z.; Sun, J.; Chen, P.; Harnly, J. UHPLC-PDA-ESI/HRMS/MS(n) analysis of anthocyanins, flavonol glycosides, and hydroxycinnamic acid derivatives in red mustard greens (Brassica juncea Coss variety). J. Agric. Food Chem. 2011, 59, 12059-12072.

108. Olsen, H.; Aaby, K.; Borge, G.I.A. Characterization and Quantification of Flavonoids and Hydroxycinnamic Acids in Curly Kale (Brassica oleracea L. Convar. acephala Var. sabellica) by HPLC-DAD-ESI-MSn. J. Agric. Food Chem. 2009, 57, 2816-2825.

109. Oszmiański, J.; Kolniak-Ostek, J.; Wojdyło, A. Characterization and Content of Flavonol Derivatives of Allium ursinum L. Plant. J. Agric. Food Chem. 2013, 61, 176-184.

110. da Costa César, I.; Braga, F.C.; Soares, C.D.V.; de Aguiar Nunan, E.; Pianetti, G.A.; Condessa, F.A.; Barbosa, T.A.F.; Campos, L.M.M. Development and validation of a RPHPLC method for quantification of isoflavone aglycones in hydrolyzed soy dry extracts. Journal of Chromatography B 2006, 836, 74-78.

111. Hertog, M.G.L.; Hollman, P.C.H.; Venema, D.P. Optimization of a quantitative HPLC determination of potentially anticarcinogenic flavonoids in vegetables and fruits. Journal of Agricultural and Food Chemistry 1992, 40, 1591-1598. 